\title{
Wilson loops and the geometry of matrix models in $\mathrm{AdS}_{4} / \mathrm{CFT}_{3}$
}

\section{Daniel Farquet and James Sparks}

Mathematical Institute, University of Oxford, 24-29 St Giles', Oxford OX1 3LB, U.K.

E-mail: farquet@maths.ox.ac.uk, sparks@maths.ox.ac.uk

ABSTRACT: We study a general class of supersymmetric $\mathrm{AdS}_{4} \times Y_{7}$ solutions of M-theory that have large $N$ dual descriptions as $\mathcal{N}=2$ Chern-Simons-matter theories on $S^{3}$. The Hamiltonian function $h_{M}$ for the M-theory circle, with respect to a certain contact structure on $Y_{7}$, plays an important role in the duality. We show that an M2-brane wrapping the M-theory circle, giving a fundamental string in $\mathrm{AdS}_{4}$, is supersymmetric precisely at the critical points of $h_{M}$, and moreover the value of this function at the critical point determines the M2-brane action. Such a configuration determines the holographic dual of a BPS Wilson loop for a Hopf circle in $S^{3}$, and leads to an effective method for computing the Wilson loop on both sides of the correspondence in large classes of examples. We find agreement in all cases, including for several infinite families, and moreover we find that the image $h_{M}\left(Y_{7}\right)$ determines the range of support of the eigenvalues in the dual large $N$ matrix model, with the critical points of $h_{M}$ mapping to points where the derivative of the eigenvalue density is discontinuous.

Keywords: Gauge-gravity correspondence, Wilson, 't Hooft and Polyakov loops, Duality in Gauge Field Theories, AdS-CFT Correspondence

ARXIV EPRINT: 1304.0784 


\section{Contents}

1 Introduction and summary 1

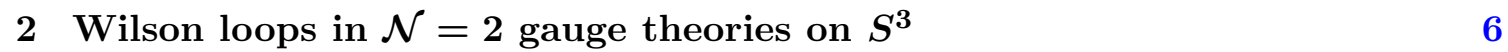

2.1 The Wilson loop 6

$\begin{array}{lll}2.2 & \text { Localization in the matrix model } & 7\end{array}$

3 BPS M2-branes 10

$\begin{array}{lll}3.1 & \text { Supergravity backgrounds } & 10\end{array}$

$\begin{array}{lll}3.2 & \text { Choice of M-theory circle } & 12\end{array}$

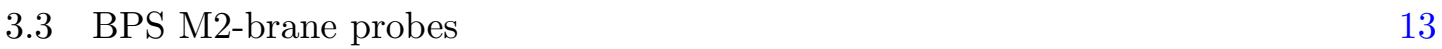

$\begin{array}{lll}3.4 & \text { M-theory hamiltonian function } & 15\end{array}$

$\begin{array}{ll}3.5 & \text { Geometric methods of computation } \\ 3.6 & 17\end{array}$

3.6 Hamiltonian function and density 20

4 Examples $\quad 21$

4.1 Duals to the round $S^{7} \quad 21$

4.1.1 ABJM theory 21

4.1.2 Mirror theory 23

$\begin{array}{ll}4.2 & \text { Dual to } Q^{1,1,1} / \mathbb{Z}_{k}\end{array} 25$

$4.3 \mathcal{N}=8$ super-Yang-Mills with flavour $\quad 26$

$4.4 L^{a, 2 a, a}$ Chern-Simons-quivers 30

4.5 $L^{a, b, a}$ Chern-Simons-quivers 32

4.6 Duals to non-Einstein solutions with flux 35

5 Discussion $\quad 36$

\section{Introduction and summary}

Over the last few years our understanding of the $\mathrm{AdS}_{4} / \mathrm{CFT}_{3}$ correspondence, particularly in M-theory, has improved considerably. Broadly speaking, this has involved developments on two fronts. Firstly, we now have large classes of very explicit examples of dual pairs; that is, gravity backgrounds for which we have some precise description of the dual superconformal field theories. Secondly, there are new quantitative tests of these conjectured dualities, based on supersymmetric localization in the field theories. The aim of this article is to extend this quantitative analysis further, by examining the computation of certain BPS Wilson loops on both sides of the correspondence. In the process we will also understand how other structures are related via the duality. 
Starting with the seminal work of [1] we now have large classes of supersymmetric $\mathrm{AdS}_{4} \times Y_{7}$ gravity backgrounds of M-theory that are associated with particular $(2+1)$ dimensional supersymmetric gauge theories, typically Chern-Simons theories coupled to matter, that are believed to have a dual superconformal fixed point. The construction of the UV gauge theory usually relies on a dual description in terms of type IIA string theory, which in turn involves a choice of M-theory circle $\mathrm{U}(1)_{M}$ acting on $Y_{7}$; different choices can lead to different UV gauge theories that flow to the same IR superconformal fixed point. In [1] the highly supersymmetric case where $Y_{7}=S^{7} / \mathbb{Z}_{k}$, equipped with its round Einstein metric and with $N$ units of flux through this internal space, was related to a large $N$ dual description as an $\mathcal{N}=6$ superconformal $\mathrm{U}(N) \times \mathrm{U}(N)$ Chern-Simons-matter theory (the ABJM theory), with $k \in \mathbb{Z}$ being the Chern-Simons coupling. Here $\mathbb{Z}_{k} \subset \mathrm{U}(1)_{M}$, with the M-theory circle action by $\mathrm{U}(1)_{M}$ being the Hopf action on $S^{7}$, so that $S^{7} / \mathrm{U}(1)_{M}=\mathbb{C P}^{3}$. There are now many families of examples of a similar type [2]-[18], generally with $\mathcal{N} \geq 2$ supersymmetry, in which $Y_{7}$ is a Sasaki-Einstein seven-manifold and the dual description typically involves supersymmetric Chern-Simons-matter theories whose gauge groups are products of unitary groups, and with matter in various representations (bifundamental, fundamental, adjoint). There are also examples in which $\mathrm{AdS}_{4} \times Y_{7}$ is a warped product, with non-trivial four-form flux on non-Einstein $Y_{7}$ (obtained thus far either by marginal [19] or relevant [20]-[25] deformations of Einstein examples).

Quantitative tests of these conjectured dualities arise by putting the (Euclidean) field theories on a compact three-manifold. The simplest case, in which this three-manifold is taken to be $S^{3}$ equipped with its round metric, was studied in [26-28]. This can be done for a completely general $\mathcal{N}=2$ supersymmetric gauge theory, in such a way to preserve supersymmetry. Moreover, using a standard argument [29] one can show that the path integral, with any BPS operator inserted, reduces exactly to a finite-dimensional matrix integral. This implies that the VEVs of BPS operators may be computed exactly using a matrix model description, with the large $N$ limit of this then expected to reproduce certain supergravity results. In practice this has been used to compute the free energy $F$ (minus the logarithm of the partition function) on both sides of the correspondence [30]-[37], where on the supergravity side this is proportional to $N^{3 / 2}$ with a coefficient depending only on the volume of $Y_{7} \cdot{ }^{1}$

It is natural to try to extend these results further, by inserting non-trivial BPS operators into the path integral, computing the corresponding large $N$ behaviour in the matrix model, and comparing to an appropriate dual semi-classical supergravity computation. In the original papers on the ABJM theory [26, 30, 38-41] the supersymmetric Wilson loop for the gauge field around a Hopf circle $S^{1} \subset S^{3}$ was studied. This is $1 / 2$ BPS, and is readily computed in the large $N$ matrix model $[26,30]$. Generally speaking, one expects such a Wilson loop to be dual to a fundamental string when viewed from a type IIA perspective [42], with the Euclidean string worldsheet having boundary on the Hopf $S^{1}$ at conformal infinity. Semi-classically, more precisely this will be a supersymmetric minimal

\footnotetext{
${ }^{1}$ For a general $\mathrm{AdS}_{4} \times Y_{7}$ solution this is the contact volume of $Y_{7}$, rather than the Riemannian volume, as we shall review in section 3 .
} 
surface $\Sigma_{2}$ in Euclidean $\mathrm{AdS}_{4}$, with the VEV calculated via the regularized area of the string worldsheet. Such a string must then be pointlike in the internal space, and for the ABJM theory this is $\mathbb{C P}^{3}=S^{7} / \mathrm{U}(1)_{M}$. Equivalently, this IIA string lifts to an M2-brane wrapping the M-theory circle. Notice that since $\mathbb{C P}^{3}$ is a homogeneous space all positions for the IIA string are equivalent. The two computations (large $N$ matrix model and area) of course agree. ${ }^{2}$

This Wilson loop is $1 / 2$ BPS in a general $\mathcal{N}=2$ supersymmetric gauge theory on $S^{3}$, as we review in section 2 , and can be computed using the large $N$ matrix model description. The supergravity dual computation will naturally involve an M2-brane wrapping the Mtheory circle, leading to the same fundamental string configuration in Euclidean $\mathrm{AdS}_{4}$ (see figure 1). The only issue is which copy of the M-theory circle is relevant? When the internal space is $Y_{7}=S^{7} / \mathbb{Z}_{k}$ all choices are equivalent by symmetry, but on a general Sasaki-Einstein manifold $Y_{7}$, or a more general non-Einstein $Y_{7}$ with flux, this is clearly not the case. Equivalently we may ask which IIA fundamental strings in $\operatorname{AdS}_{4} \times M_{6}$, that are pointlike in $M_{6}=Y_{7} / \mathrm{U}(1)_{M}$, preserve any supersymmetry.

Summary of results. Our starting point is to consider BPS M2-branes in general $\mathcal{N}=2$ supersymmetric $\mathrm{AdS}_{4} \times Y_{7}$ solutions of eleven-dimensional supergravity. These backgrounds were studied in detail in $[24,25]$, where it was shown that provided the quantized M2-brane charge $N$ of the background (measured by a certain flux integral) is non-zero, then there is always a canonical contact one-form $\eta$ defined on $Y_{7}$. Concretely, $\eta$ is constructed as a bilinear in the Killing spinors on $Y_{7}$, and it was shown in the latter reference that this contact structure entirely captures both the gravitational free energy of the background, and also the scaling dimensions of BPS operators arising from supersymmetric M5-branes wrapped on five-manifolds $\Sigma_{5} \subset Y_{7}$.

In this paper we will show that the same contact form $\eta$ captures the Wilson loop VEV $\langle W\rangle$ of interest, computed semi-classically from the action of a BPS M2-brane. Concretely, we derive the general formula

$$
\log \langle W\rangle_{\text {gravity }}=\frac{(2 \pi)^{2} \int_{S_{M}^{1}} \eta}{\sqrt{96 \operatorname{Vol}_{\eta}\left(Y_{7}\right)}} N^{1 / 2},
$$

where we have defined the contact volume of $Y_{7}$ as

$$
\operatorname{Vol}_{\eta}\left(Y_{7}\right) \equiv \frac{1}{48} \int_{Y_{7}} \eta \wedge(\mathrm{d} \eta)^{3}
$$

In particular, a supersymmetric M2-brane is calibrated with respect to $\eta$, which is why the integral of $\eta$ along the M-theory circle $S_{M}^{1}$ appears in the formula (1.1). A contact form $\eta$ always has an associated unique Reeb vector field $\xi$, defined via the equations $\xi\lrcorner \eta=1$, $\xi\lrcorner \mathrm{d} \eta=0$, and in $[24,25]$ it was shown that $\xi$ is also the $R$-symmetry Killing vector field,

\footnotetext{
${ }^{2}$ Similar Wilson loops have recently been considered in five-dimensional superconformal field theories on $S^{5}$ [43], which may also be computed using localization techniques. The gravity duals are described by warped $\mathrm{AdS}_{6} \times S^{4} / \mathbb{Z}_{n}$ solutions of massive IIA supergravity, and thus the geometry of the internal spaces here is fixed and in fact unique [44].
} 


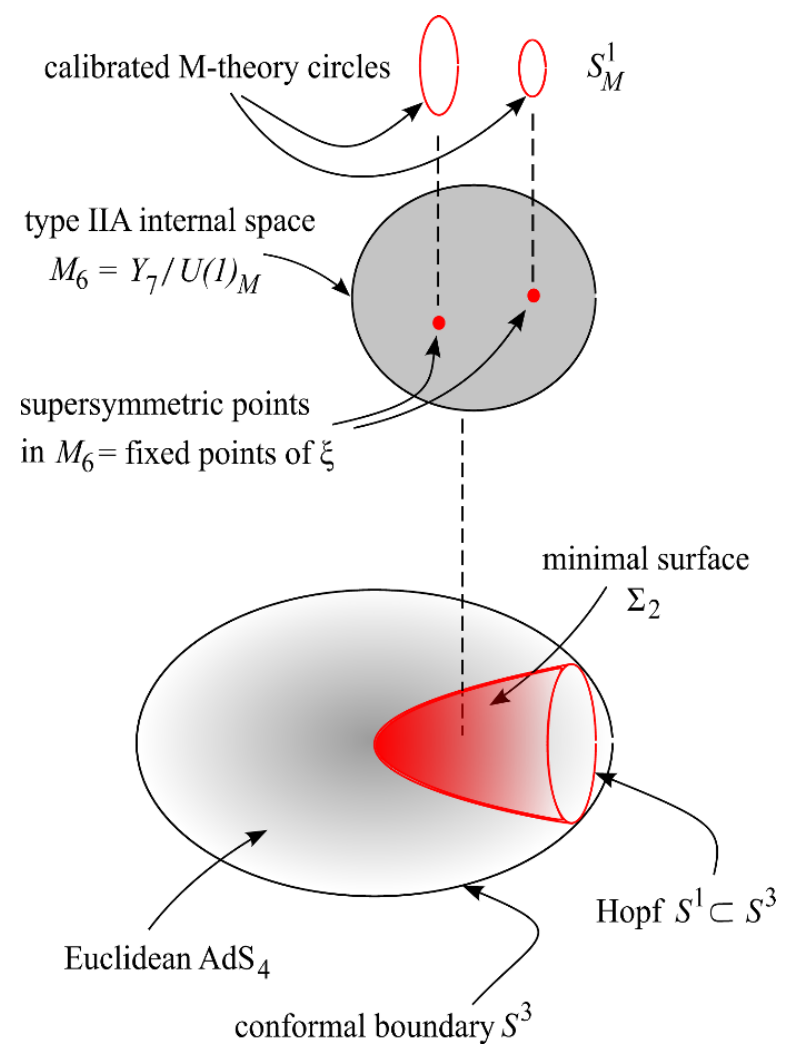

Figure 1. A depiction of the total spacetime $\mathrm{AdS}_{4} \times Y_{7}$, with a choice of M-theory circle $\mathrm{U}(1)_{M}$, together with the supersymmetric M2-branes of interest which are shown in red. These M2-branes are pointlike in the type IIA internal space $M_{6}=Y_{7} / \mathrm{U}(1)_{M}$, wrapping copies of the M-theory circle over these points, and are calibrated by the contact form $\eta$. The supersymmetric points in $M_{6}$ are precisely the points where the projection of the R-symmetry/Reeb vector field $\xi$ is zero (giving fixed points on $M_{6}$ ), and in general the calibrated circles over such points have different lengths. The remaining worldvolume of the M2-brane wraps a minimal supersymmetric surface $\Sigma_{2}$ in Euclidean $\mathrm{AdS}_{4}$. The latter may be viewed as a hyperbolic 4-ball, with conformal boundary $S^{3}$, and $\Sigma_{2}$ then has the topology of a 2-ball, with boundary a Hopf $S^{1} \subset S^{3}$.

that is expected since an $\mathcal{N}=2$ superconformal theory in three dimensions has a $\mathrm{u}(1)_{R}$ symmetry in the superconformal algebra. We will show that an M2-brane wrapping a copy of the M-theory circle $S_{M}^{1}$ is supersymmetric precisely when the generating vector field $\zeta_{M}$ of $\mathrm{U}(1)_{M}$ is proportional to $\xi$. Geometrically, this means that the corresponding fundamental string at a point $p \in M_{6}$ is supersymmetric precisely when $p$ is a fixed point of $\xi$, considered as a vector field on $M_{6}$ (on $Y_{7}$, on the other hand, $\xi$ is always nowhere zero).

There is another way to describe which wrapped M2-branes are supersymmetric which involves the Hamiltonian function for the M-theory circle, defined as

$$
h_{M} \equiv \eta\left(\zeta_{M}\right) .
$$

This is a real function $h_{M}: Y_{7} \rightarrow \mathbb{R}$, invariant under $\zeta_{M}$, and we show that the supersymmetric M-theory circles $S_{M}^{1} \subset Y_{7}$ lie precisely on the critical set $\mathrm{d} h_{M}=0$. The action of a 
supersymmetric M2-brane corresponding to a point $p \in M_{6}$ may then also be written as

$$
-S_{\mathrm{M} 2}=\frac{(2 \pi)^{3} h_{M}(\hat{p})}{\sqrt{96 \operatorname{Vol}_{\eta}\left(Y_{7}\right)}} N^{1 / 2},
$$

where $\hat{p} \in Y_{7}$ is any point that projects to $p \in M_{6}=Y_{7} / \mathrm{U}(1)_{M}$. Since (1.4) depends only on $\eta$ we may compute this expression in examples using the same methods employed in $[24,25,45]-[49]$. For example, for toric solutions (1.4) may be computed entirely using toric geometry methods. In general there are multiple supersymmetric $S_{M}^{1}$ circles, which can have different lengths with respect to $\eta$ and thus leading to different actions (1.4). In the semi-classical computation one should sum over all such configurations, which in the large $N$ limit then implies that in (1.1) it is the longest $S_{M}^{1}$ that gives the leading contribution to the Wilson loop.

In the families of examples that we shall study, the dual field theory computation of the Wilson loop VEV reduces to a computation in a large $N$ matrix model. As we shall review in section 2, in this matrix model the eigenvalues at large $N$ take the general form $\lambda^{I}=x N^{1 / 2}+\mathrm{i} y^{I}(x)$, where the index $I$ runs over the number of factors of $\mathrm{U}(N)$ in the gauge group $G=\prod_{I} \mathrm{U}(N)$, and are described by an eigenvalue density function $\rho(x)$ which is supported on some interval $\left[x_{\min }, x_{\max }\right] \subset \mathbb{R}$. To leading order at large $N$ it is straightforward to compute

$$
\log \langle W\rangle_{\mathrm{QFT}}=x_{\max } N^{1 / 2},
$$

which should be compared to the dual supergravity result (1.1).

Remarkably, in all examples that we study we find that the interval $\left[x_{\min }, x_{\max }\right]$ in the matrix model coincides, in a precise way, with the image of the Hamiltonian function $h_{M}\left(Y_{7}\right)$. Since $Y_{7}$ is compact and connected, the latter image is also necessarily a closed interval, and more precisely we find $h_{M}\left(Y_{7}\right)=\left[c_{\min }, c_{\max }\right]$, where the field theory quantity $x$ is proportional to the geometrical quantity $c$ :

$$
x=\frac{(2 \pi)^{3}}{\sqrt{96 \mathrm{Vol}_{\eta}\left(Y_{7}\right)}} c .
$$

The Hamiltonian $h_{M}$ is a Morse-Bott function on the symplectic cone over $Y_{7}$, and on general grounds we know that the image interval $\left[c_{\min }, c_{\max }\right]$ is divided into $P$ subintervals $c_{\min }=c_{1}<c_{2}<\cdots<c_{P+1}=c_{\max }$, where the critical set maps as $h_{M}\left(\left\{\mathrm{~d} h_{M}=0\right\}\right)=$ $\left\{c_{i} \mid i=1, \ldots, P+1\right\}$. For all $c \in\left(c_{i}, c_{i+1}\right)$ the level surfaces $h_{M}^{-1}(c) \subset Y_{7}$ are diffeomorphic to a fixed six-manifold, with the topology changing precisely as one passes a critical point $c_{i}$. Even more remarkable is that we find that the corresponding points $x_{i}$, related to $c_{i}$ via (1.6), are precisely the points where $\rho^{\prime}(x)$ has a jump discontinuity in the matrix model. These points are then also related to the fixed points of the Reeb vector $\xi$ on $M_{6}$.

The outline of the rest of this article is as follows. In section 2 we review the definition of the BPS Wilson loop in $\mathcal{N}=2$ Chern-Simons-matter theories, and how it may be computed in the large $N$ matrix model. Section 3 analyses supersymmetric M2-branes in a general class of $\mathrm{AdS}_{4} \times Y_{7}$ backgrounds in M-theory, and we derive the general formula for the 
action (1.4), leading to the holographic Wilson loop result (1.1). In section 4 we compute the Wilson loop, on both sides of the correspondence, in a variety of examples, including for several infinite families of Sasaki-Einstein $Y_{7}$, and for models with non-Einstein $Y_{7}$. Section 5 concludes with a brief discussion.

\section{Wilson loops in $\mathcal{N}=2$ gauge theories on $S^{3}$}

The dual superconformal field theories of interest have UV descriptions as $\mathcal{N}=2$ ChernSimons gauge theories with matter on $S^{3}$. We begin in this section by defining the BPS Wilson loop in such a theory, summarize how it localizes in the matrix model, and explain how it can be efficiently calculated. This section is mainly a review of material already in the literature.

\subsection{The Wilson loop}

In $\mathcal{N}=2$ supersymmetric gauge theories the gauge field $A_{\mu}$ is part of a vector multiplet that also contains two real scalars $\sigma$ and $D$, that are auxiliary fields, and a two-component spinor $\lambda$, all of which are in the adjoint representation of the gauge group $G$. The BPS Wilson loop in a representation $\mathcal{R}$ of $G$ is given by

$$
W=\frac{1}{\operatorname{dim} \mathcal{R}} \operatorname{Tr}_{\mathcal{R}}\left[\mathcal{P} \exp \left(\oint_{\gamma} \mathrm{d} s\left(\mathrm{i} A_{\mu} \dot{x}^{\mu}+\sigma|\dot{x}|\right)\right)\right],
$$

where $x^{\mu}(s)$ parametrizes the worldline $\gamma \subset S^{3}$ of the Wilson line and the path ordering operator has been denoted by $\mathcal{P}$. For a Chern-Simons theory the gauge multiplet has a kinetic term described by the supersymmetric Chern-Simons action

$$
S_{\text {Chern-Simons }}=\frac{k}{4 \pi} \int \mathrm{d}^{3} x \sqrt{\operatorname{det} g} \operatorname{Tr}\left(A \wedge \mathrm{d} A+\frac{2}{3} A \wedge A \wedge A-\lambda^{\dagger} \lambda+2 D \sigma\right),
$$

where here $g$ is the round metric on $S^{3}$, and $k$ denotes the Chern-Simons coupling. When $G$ is a product of unitary groups, $G=\prod_{I} \mathrm{U}\left(N_{I}\right)$, one can in general take different $k_{I} \in \mathbb{Z}$ for each factor. In this case we will denote $k=\operatorname{gcd}\left\{k_{I}\right\}$ [3].

There are four Killing spinors on $S^{3}$, two satisfying each choice of sign in the equation $\nabla_{\mu} \varepsilon= \pm \frac{\mathrm{i}}{2} \tau_{\mu} \varepsilon$, where the gamma matrices $\tau_{\mu}$ in an orthonormal frame generate the Clifford algebra Cliff $(3,0)$, and may thus be taken to be the Pauli matrices. A natural orthonormal frame $\left\{e^{m}\right\}_{m=1,2,3}$ on $S^{3}$ is provided by the left (or right) invariant one-forms under the isometry group $\mathrm{SU}(2)_{\text {left }} \times \mathrm{SU}(2)_{\text {right }}$. The four Killing spinors on $S^{3}$ transform in the $(\mathbf{2}, \mathbf{1}),(\mathbf{1}, \mathbf{2})$ representations of this group.

The full supersymmetry transformations for a vector multiplet and matter multiplet may be found in [26-28]. For our purposes we need note only that localization of the path integral, discussed in the next section, requires one to choose a Killing spinor $\varepsilon$, which without loss of generality we assume solves $\nabla_{\mu} \varepsilon=\frac{\mathrm{i}}{2} \tau_{\mu} \varepsilon$. This choice of Killing spinor then has the two associated supersymmetry transformations

$$
\begin{aligned}
\delta A_{\mu} & =-\frac{\mathrm{i}}{2} \lambda^{\dagger} \tau_{\mu} \varepsilon, \\
\delta \sigma & =-\frac{1}{2} \lambda^{\dagger} \varepsilon .
\end{aligned}
$$


If one varies the Wilson loop (2.1) under the latter supersymmetry transformation one obtains

$$
\delta W \propto \frac{1}{2} \lambda^{\dagger}\left(\tau_{\mu} \dot{x}^{\mu}-|\dot{x}|\right) \varepsilon
$$

The Wilson loop is then invariant under supersymmetry provided

$$
\left(\tau_{\mu} \dot{x}^{\mu}-|\dot{x}|\right) \varepsilon=0 \text {. }
$$

Choosing $s$ to parametrize arclength, so that $|\dot{x}|=1$ along the loop, we see that $\tau_{\mu} \dot{x}^{\mu}$ must be constant. In the left-invariant orthonormal frame $e^{m}$ one may then align $\dot{x}^{\mu}$ along one direction, say $e^{3}$. The integral curve of this vector field is a Hopf $S^{1} \subset S^{3}$ (or equivalently a great circle). The supersymmetry condition then becomes

$$
\left(\tau_{3}-1\right) \varepsilon=0 \text {. }
$$

This projection condition then fixes one of the two possible choices of $\varepsilon$ satisfying $\nabla_{\mu} \varepsilon=$ $\frac{\mathrm{i}}{2} \tau_{\mu} \varepsilon$, implying that the Wilson loop (2.1) is indeed a $1 / 2$ BPS operator provided one takes $\gamma$ to be a Hopf circle. We will see later on that the condition (2.6), plus the fact that the supersymmetry generators are Killing spinors, also arises as the condition for supersymmetry of a probe M2-brane.

\subsection{Localization in the matrix model}

The VEV of the BPS Wilson loop (2.1) is, by definition, obtained by inserting $W$ into the path integral for the theory on $S^{3}$. The computation of this is greatly simplified by the fact that this path integral localizes onto supersymmetric configurations of fields. We summarize the main steps and results in this section, following in particular [26, 27, 30, 31], and refer the reader to the original papers for further details.

The central idea is that the path integral, with $W$ inserted, is invariant under the supersymmetry variation $\delta$ corresponding to the Killing spinor $\varepsilon$ satisfying (2.6). We have written two of the supersymmetry variations in (2.3), and the variations of other fields (including fields in the chiral matter multiplets) may be found in the above references. Crucially, $\delta^{2}=0$ is nilpotent. There is then a form of fixed point theorem that implies that the only net contributions to this path integral come from field configurations that are invariant under $\delta$ [50]. Formally, one can argue this by introducing a collective Grassmann coordinate $\vartheta$ along the direction defined by $\delta$ in field space, and then appeal to the fact that the Grassmann integral $\int \mathrm{d} \vartheta=0$. This then breaks down precisely at fixed points of $\delta$, where the coordinate $\vartheta$ is not defined.

Alternatively, and more practically for computation, one may add a conveniently chosen $\delta$-exact positive definite term to the action, which a standard argument shows does not affect the expectation value of any supersymmetric ( $\delta$-invariant) operator. For the vector multiplet one can add the term $t \operatorname{Tr}\left[(\delta \lambda)^{\dagger} \delta \lambda\right]$ to the action (a similar term exists for a matter multiplet), without affecting the path integral. Sending $t \rightarrow \infty$ one notes that, due to the form of this term added to the Lagrangian, only configurations with $\delta \lambda=0$ contribute to the path integral in a saddle point approximation. This saddle point then 
gives the same value as if the path integral had been calculated with $t=0$, which is the quantity we are interested in. The saddle point approximation requires one to compute a one-loop determinant around the $\delta$-invariant field configurations, which in the terminology of fixed point theorems is the contribution from the normal bundle to the fixed point set in field space.

For the $\mathcal{N}=2$ supersymmetric Chern-Simons-matter theories of interest, one finds that the $\delta$-invariant configurations on $S^{3}$ are particularly simple:

$$
A_{\mu}=0, \text { and } D=-\sigma=\text { constant }
$$

with all fields in the matter multiplet set identically to zero. Here we may diagonalize $\sigma$ by a gauge transformation. For a $\mathrm{U}(N)$ gauge group we may thus write $\sigma=\operatorname{diag}\left(\frac{\lambda_{1}}{2 \pi}, \ldots \frac{\lambda_{N}}{2 \pi}\right)$, thus parametrizing $2 \pi \sigma$ by its eigenvalues $\lambda_{i}$. The theories of interest will have a product gauge group of the form $G=\prod_{I=1}^{g} \mathrm{U}(N)$, and for $t=\infty$ the partition function then takes the saddle point form

$$
Z=\frac{1}{(N !)^{g}} \int\left(\prod_{I=1}^{g} \prod_{i=1}^{N} \frac{\mathrm{d} \lambda_{i}^{I}}{2 \pi}\right) \exp \left[\mathrm{i} \sum_{I=1}^{g} \frac{k_{I}}{4 \pi} \sum_{i=1}^{N}\left(\lambda_{i}^{I}\right)^{2}\right] \mathrm{e}^{-F_{\text {one-loop }}}
$$

where the one-loop determinant is given by

$$
\mathrm{e}^{-F_{\text {one-loop }}}=\prod_{I=1}^{g} \prod_{i \neq j} 2 \sinh \frac{\lambda_{i}^{I}-\lambda_{j}^{I}}{2} \cdot \prod_{\text {matter } \alpha} \operatorname{det}_{\mathcal{R}_{\alpha}} \exp \left[\ell\left(1-\Delta_{\alpha}+\mathrm{i} \sigma\right)\right] .
$$

Here the first exponential term in (2.8) is simply the classical Chern-Simons action in (2.2), evaluated on the localized constant field configuration (2.7). The one-loop determinant factorizes, and the first term in (2.9) is the one-loop determinant for the vector multiplet. Since we have used gauge-invariance in (2.8) to restrict the integral to the Cartan subalgebra, we also have a Vandermonde determinant which has been cancelled against a term that appears in the one-loop determinant. The second term in (2.9) involves a product over chiral matter multiplets, labelled by $\alpha$. We have taken the $\alpha^{\text {th }}$ multiplet to be in representation $\mathcal{R}_{\alpha}$, and with R-charge $\Delta_{\alpha}$. The determinant in the representation $\mathcal{R}_{\alpha}$ is understood to be a product over weights $\varrho$ in the weight-space decomposition of this representation, and $\sigma$ is then understood to mean $\varrho(\sigma)$ in (2.9). Finally,

$$
\ell(z)=-z \log \left(1-\mathrm{e}^{2 \pi \mathrm{i} z}\right)+\frac{\mathrm{i}}{2}\left[\pi z^{2}+\frac{1}{\pi} \operatorname{Li}_{2}\left(\mathrm{e}^{2 \pi \mathrm{i} z}\right)\right]-\frac{\mathrm{i} \pi}{12} .
$$

In this set-up, the VEV of the BPS Wilson loop (2.1) reduces to

$$
\langle W\rangle=\frac{1}{Z(N !)^{g} \operatorname{dim} \mathcal{R}} \int\left(\prod_{I=1}^{g} \prod_{i=1}^{N} \frac{\mathrm{d} \lambda_{i}^{I}}{2 \pi}\right) \mathrm{e}^{\mathrm{i} \sum_{I=1}^{g} \frac{k_{I}}{4 \pi} \sum_{i=1}^{N}\left(\lambda_{i}^{I}\right)^{2}} \operatorname{Tr}_{\mathcal{R}}\left(\mathrm{e}^{2 \pi \sigma}\right) \mathrm{e}^{-F_{\text {one-loop }}} .
$$

Notice the integrand is the same as that for the partition function (2.8), with an additional insertion of $\operatorname{Tr}_{\mathcal{R}}\left(\mathrm{e}^{2 \pi \sigma}\right)$ arising from the Wilson loop operator. The factor of $(N !)^{g}$, as in (2.8), arises from dividing by residual Weyl transformations, which for $\mathrm{U}(N)$ introduces 
a factor of $1 / N$ !. Note also that we have normalized the VEV relative to the partition function $Z$, so that $\langle 1\rangle=1$, as is usual in quantum field theory.

Localization has reduced the partition function $Z$ and the Wilson loop VEV to finitedimensional integrals (2.8), (2.11) over the eigenvalues $\lambda_{i}^{I}$ of $\sigma$, but in practice these are difficult to evaluate explicitly due to the complicated one-loop effective potential (2.9). For comparison to the dual supergravity results we must take the $N \rightarrow \infty$ limit, where the number of eigenvalues, and hence integrals, tends to infinity. One can then attempt to compute this limit using a saddle point approximation of the integral (this is then our second application of the saddle point method). With the exception of the $\mathcal{N}=6$ supersymmetric ABJM theory, where this matrix model is well-understood [51], for general $\mathcal{N}=2$ theories the large $N$ limit of the matrix integrals is not understood rigorously. However, in [30] a simple ansatz for the large $N$ limit of the saddle point eigenvalue distribution was introduced. This ansatz is based on a partial analytic analysis of the matrix model, and also on a numerical approach to computing the saddle point. One seeks saddle points with eigenvalues of the form

$$
\lambda_{i}^{I}=x_{i} N^{\beta}+\mathrm{i} y_{i}^{I}
$$

with $x_{i}$ and $y_{i}^{I}$ real and assumed to be $\mathcal{O}(1)$ in a large $N$ expansion, and $\beta>0$. In the large $N$ limit the real part is assumed to become dense. Ordering the eigenvalues so that the $x_{i}$ are strictly increasing, the real part becomes a continuous variable $x$, with density $\rho(x)$, while $y_{i}^{I}$ becomes a continuous function of $x, y^{I}(x)$.

Substituting this ansatz into the partition function expression (2.8), the sums over eigenvalues become Riemann integrals over $x$, and one finds that the double sums appearing in the one-loop expression (2.9) effectively have a delta function contribution which reduces them to single integrals over $x$. (This is often described by saying that the long range forces in the matrix model cancel.) Writing $Z=\mathrm{e}^{-F}$ one then obtains a functional $F\left[\rho(x), y^{I}(x)\right]$, with $x$ supported on some interval $\left[x_{\min }, x_{\max }\right]$, and to apply the saddle point method one then extremizes $F$ with respect to $\rho(x), y^{I}(x)$, subject to the constraint that $\rho(x)$ is a density

$$
\int_{x_{\min }}^{x_{\max }} \rho(x) \mathrm{d} x=1
$$

The existence of such a saddle point fixes the exponent $\beta=\frac{1}{2}$ in (2.12). One then finally also extremizes over the choice of interval, by varying with respect to $x_{\min }, x_{\max }$, to obtain the saddle point eigenvalue distribution $\rho(x), y^{I}(x)$.

We shall be interested in evaluating the Wilson loop VEV (2.11) in the fundamental representation, so that the Wilson loop is proportional to $\sum_{I=1}^{g} \sum_{i=1}^{N} \mathrm{e}^{\lambda_{i}^{I}}$. In the large $N$ limit, described by the saddle point density $\rho(x)$ and imaginary parts $y^{I}(x)$ of the eigenvalues, the VEV reduces simply to

$$
\langle W\rangle_{\mathrm{QFT}}=N \sum_{I=1}^{g} \int_{x_{\min }}^{x_{\max }} \mathrm{e}^{x N^{1 / 2}+\mathrm{i} y^{I}(x)} \rho(x) \mathrm{d} x .
$$


Because of the form of $F\left[\rho(x), y^{I}(x)\right]$ for $\mathcal{N}=2$ Chern-Simons-matter theories, the saddle point eigenvalue density $\rho(x)$ is always a continuous, piecewise linear function on $\left(x_{\min }, x_{\max }\right)$. A simple computation then shows that, to leading order in the large $N$ limit, the matrix model VEV (2.14) reduces to

$$
\log \langle W\rangle_{\mathrm{QFT}}=x_{\max } N^{1 / 2} .
$$

This is our final formula for the large $N$ limit of the Wilson loop VEV. We see that it computes the maximum value of the (real part of the) saddle point eigenvalues.

In our summary above we have suppressed the dependence on the R-charges $\Delta_{\alpha}$ of the matter multiplets, labelled by $\alpha$, appearing in (2.9). If these are left arbitrary, one obtains a free energy $F$ that is a function of $\Delta_{\alpha}$, and according to [27] the superconformal R-symmetry of an $\mathcal{N}=2$ superconformal field theory further extremizes $F$ as a function of $\Delta_{\alpha}$ (in fact maximizing $F[52]$ ). For theories with M-theory duals of the form $\mathrm{AdS}_{4} \times Y_{7}$ one finds the expected supergravity result

$$
F=\sqrt{\frac{2 \pi^{6}}{27 \mathrm{Vol}_{\eta}\left(Y_{7}\right)}} N^{3 / 2},
$$

but as a function of R-charges $\Delta_{\alpha}$ [31], where on the right hand side it is in general the contact volume (1.2) of $Y_{7}$ that appears, as a function of the Reeb vector field $\xi$. This has by now been demonstrated in many classes of examples in the literature [25, 30]-[37].

\section{BPS M2-branes}

In this section we analyse the supersymmetric probe M2-branes that are relevant for computing the holographic dual of the Wilson loop VEV (2.15). We first recast the condition of supersymmetry into a geometric condition, then derive the formula (1.4) for the action of the M2-brane, and finally describe how this may be computed in practice using different geometric methods.

\subsection{Supergravity backgrounds}

We will study the general class of $\mathcal{N}=2$ supersymmetric $\mathrm{AdS}_{4} \times Y_{7}$ backgrounds of Mtheory described in $[24,25]$. We begin by recalling some relevant results and formulae. The eleven-dimensional metric and four-form $G_{4}$ take the form

$$
\begin{aligned}
g_{11} & =\mathrm{e}^{2 \Delta}\left(\frac{1}{4} g_{\mathrm{AdS}_{4}}+g_{Y_{7}}\right), \\
G_{4} & =\frac{m}{16} \mathrm{vol}_{4}+F_{4},
\end{aligned}
$$

where the metric on $\mathrm{AdS}_{4}$ here has unit AdS radius, with volume form vol 4 . The warp factor $\Delta$ is taken to be a function on $Y_{7}, m$ is a constant, and $F_{4}$ is a four-form on $Y_{7}$. This is the most general ansatz compatible with the symmetries of $\mathrm{AdS}_{4}$. The eleven-dimensional Majorana spinor takes the form

$$
\epsilon=\mathrm{e}^{\Delta / 2} \psi_{+} \otimes \chi_{+}+\mathrm{e}^{\Delta / 2} \psi_{-} \otimes \chi_{-}+\text {charge conjugate },
$$


where $\chi_{ \pm}$are complex spinors on $Y_{7}, \psi_{ \pm}$are the usual Killing spinors on $\mathrm{AdS}_{4}$ (the \pm signs are related to the charge under the R-symmetry, discussed below), and the factors of $\mathrm{e}^{\Delta / 2}$ have been introduced for convenience.

In general the spinors $\chi_{ \pm}$solve quite a complicated system of coupled first order equations on $Y_{7}$, that may be found in $[24,25]$. These equations are then necessary and sufficient for supersymmetry of the $\mathrm{AdS}_{4} \times Y_{7}$ background. For our purposes we need note only a few key formulae. We first define the real one-forms

$$
\xi \equiv \mathrm{i} \bar{\chi}_{+}^{c} \gamma_{(1)} \chi_{-}, \quad \eta \equiv-\frac{6}{m} \mathrm{e}^{3 \Delta} \bar{\chi}_{+} \gamma_{(1)} \chi_{+},
$$

where in general we denote $\gamma_{(n)} \equiv \frac{1}{n !} \gamma_{m_{1} \cdots m_{n}} \mathrm{~d} y^{m_{1}} \wedge \cdots \wedge \mathrm{d} y^{m_{n}}$, with $y^{1}, \ldots, y^{7}$ local coordinates on $Y_{7}$, and the superscript $c$ on the spinors denotes charge conjugation. By an abuse of notation, we'll more generally regard $\xi$ as the dual vector field defined by the metric $g_{Y_{7}}$. We then note that the differential equations for $\chi_{ \pm}$imply the equations

$$
\begin{gathered}
\bar{\chi}_{+} \chi_{+}=\bar{\chi}_{-} \chi_{-}=1, \quad \frac{m}{6} \mathrm{e}^{-3 \Delta}=-\operatorname{Im}\left[\bar{\chi}_{+}^{c} \chi_{-}\right], \quad \operatorname{Re}\left[\bar{\chi}_{+}^{c} \chi_{-}\right]=0, \\
\operatorname{Re}\left[\bar{\chi}_{+}^{c} \gamma_{(1)} \chi_{-}\right]=0, \quad \bar{\chi}_{+} \gamma_{(1)} \chi_{+}=-\bar{\chi}_{-} \gamma_{(1)} \chi_{-}, \\
\mathrm{d} \eta=-\frac{12}{m} \mathrm{e}^{3 \Delta} \operatorname{Re}\left[\bar{\chi}_{+}^{c} \gamma_{(2)} \chi_{-}\right] .
\end{gathered}
$$

These equations may all be found in reference [25].

The one-form $\eta$ is a contact form on $Y_{7}$, meaning that the top form $\eta \wedge(\mathrm{d} \eta)^{3}$ is nowhere zero. Indeed, one finds [25] that

$$
\eta \wedge(\mathrm{d} \eta)^{3}=\frac{2^{7} 3^{4}}{m^{3}} \mathrm{e}^{9 \Delta} \mathrm{vol}_{7}
$$

where $\operatorname{vol}_{7}$ is the Riemannian volume form defined by $g_{Y_{7}}$. It is a general fact that a contact form $\eta$ has associated to it a unique Reeb vector field, defined by the relations

$$
\xi\lrcorner \eta=1, \quad \xi\lrcorner \mathrm{d} \eta=0,
$$

and remarkably one finds that $\xi$ and $\eta$ defined by (3.3) indeed satisfy these equations. Moreover, $\xi$ is a Killing vector field under which $\chi_{ \pm}$carry charges \pm 2 , and as such is the expected R-symmetry vector field.

Dirac quantization in this background implies that

$$
N=-\frac{1}{\left(2 \pi \ell_{p}\right)^{6}} \int_{Y_{7}} *{ }_{11} G_{4}+\frac{1}{2} C_{3} \wedge G_{4}
$$

should be an integer, where $\ell_{p}$ denotes the eleven-dimensional Planck length and $G_{4}=\mathrm{d} C_{3}$. This may be identified with the M2-brane charge of the background, and a computation [24, 25] gives

$$
N=\frac{1}{\left(2 \pi \ell_{p}\right)^{6}} \frac{m^{2}}{2^{5} 3^{2}} \int_{Y_{7}} \eta \wedge(\mathrm{d} \eta)^{3}
$$


relating the quantized M2-brane charge to the contact volume (1.2) of $Y_{7}$ and $m$. Since this is proportional to $m^{2}$, in fact the contact form in (3.3) may be defined only when this charge is non-zero, so that $m \neq 0$. We assume this henceforth.

The above supergravity solution of M-theory is valid only in the large $N$ limit, even for solutions with non-trivial warp factor $\Delta$ and internal four-form flux $F_{4}$. To see this [25], note that the scaling symmetry of eleven-dimensional supergravity in which the metric $g_{11}$ and four-form $G_{4}$ have weights two and three, respectively, leads to a symmetry in which one shifts $\Delta \rightarrow \Delta+\kappa$ and simultaneously scales $m \rightarrow \mathrm{e}^{3 \kappa} m, F_{4} \rightarrow \mathrm{e}^{3 \kappa} F$, where $\kappa$ is any real constant. We may then take the metric $g_{Y_{7}}$ on $Y_{7}$ to be of order $\mathcal{O}(1)$ in $N$, and conclude from the quantization condition (3.8), which has weight 6 on the right hand side, and the expression for $m \mathrm{e}^{-3 \Delta}$ in (3.1) that $\mathrm{e}^{\Delta}=\mathcal{O}\left(N^{1 / 6}\right)$. It follows that the $\mathrm{AdS}_{4}$ radius, while dependent on $Y_{7}$ in general, is $R_{\mathrm{AdS}_{4}}=\mathrm{e}^{\Delta}=\mathcal{O}\left(N^{1 / 6}\right)$, and that the supergravity approximation we have been using is valid only in the $N \rightarrow \infty$ limit.

\subsection{Choice of M-theory circle}

In addition to this background we must also pick a choice of M-theory circle. Geometrically, this means we also choose a $\mathrm{U}(1)=\mathrm{U}(1)_{M}$ action on $Y_{7}$. At first sight it might seem to be contradictory that the supergravity computation we describe then manifestly depends on a choice of M-theory circle, while the dual superconformal field theory apparently does not. However, recall that the UV description of the gauge theory, whose Lagrangian we used to compute the localized path integral and Wilson loop in section 2.2, does in fact require a choice of M-theory circle $\mathrm{U}(1)_{M}$. We may have two or more such theories, arising from different choices of $\mathrm{U}(1)_{M}$ and flowing to the same superconformal fixed point; but it does not follow that the Wilson loop operators in these theories map to each other. One thus expects the Wilson loop VEV to depend on a choice of M-theory circle, in general.

In terms of the supergravity solution described in the previous section, a choice of $\mathrm{U}(1)_{M}$ implies the choice of a (non-U(1) $R$ ) Killing vector field $\zeta_{M}$ on $\left(Y_{7}, g_{Y_{7}}\right)$, whose flow generates the M-theory circle action. In particular $\zeta_{M}$ should preserve the Killing spinors $\chi_{ \pm}$on $Y_{7}$, and hence also the contact one-form $\eta$. The type IIA spacetime is then a warped product $\mathrm{AdS}_{4} \times M_{6}$, where $M_{6} \equiv Y_{7} / \mathrm{U}(1)_{M}$ is the quotient space.

Of course globally we must be careful when writing $M_{6}=Y_{7} / \mathrm{U}(1)_{M}$. Although in principle one might choose any $\mathrm{U}(1)_{M}$ action on $Y_{7}$, in practice the gauge theories we study arise from "nice" actions of $\mathrm{U}(1)_{M}$. In particular, if the action is free then $M_{6}$ inherits the structure of a smooth manifold from $Y_{7}$, the simplest example being that of the ABJM theory with $M_{6}=\mathbb{C P}^{3}=S^{7} / \mathrm{U}(1)_{\text {Hopf }}$. If one embeds $S^{7} \subset \mathbb{C}^{4}$ as a unit sphere in the obvious way, then recall that $\mathrm{U}(1)_{\text {Hopf }}$ may be taken to have weights $(1,1,-1,-1)$ on the four complex coordinates $\left(z_{1}, z_{2}, z_{3}, z_{4}\right)$ on $\mathbb{C}^{4}$. In this case the dual field theory is the $\mathcal{N}=6$ ABJM theory, which in $\mathcal{N}=2$ language is a $\mathrm{U}(N) \times \mathrm{U}(N)$ Chern-Simons gauge theory with two chiral matter fields $A_{1}, A_{2}$ in the bifundamental $(\mathbf{N}, \overline{\mathbf{N}})$ representation of this gauge group, two chiral matter fields $B_{1}, B_{2}$ in the conjugate $(\overline{\mathbf{N}}, \mathbf{N})$ representation, and a quartic superpotential.

Another important case is when $\mathrm{U}(1)_{M}$ acts on $Y_{7}$ with a codimension four fixed point set $\mathscr{F} \subset Y_{7}$, and is free on the complement of this fixed point set. In this case the action 
on the normal space $\mathbb{R}^{4}$ to a fixed point is via $\left(w_{1}, w_{2}\right) \mapsto\left(\mathrm{e}^{\mathrm{i} \varphi} w_{1}, \mathrm{e}^{\mathrm{i} \varphi} w_{2}\right)$, where locally $\zeta_{M}=\partial_{\varphi}$ and $\left(w_{1}, w_{2}\right)$ are complex coordinates on $\mathbb{R}^{4}=\mathbb{C} \oplus \mathbb{C}$. In this case the quotient normal space is $\mathbb{R}^{3}=\mathbb{R}^{4} / \mathrm{U}(1)_{M}$, with the fixed point set $\mathscr{F}$ at the origin becoming a D6-brane locus in the type IIA spacetime. With this understanding, the IIA spacetime is again a smooth $\mathrm{AdS}_{4} \times M_{6}$, but with an $\mathrm{AdS}_{4}$-filling D6-brane wrapping $\mathscr{F}$, now thought of as a submanifold $\mathscr{F} \subset M_{6}$. Again, the simplest example is a quotient of $S^{7}$, but now where $\mathrm{U}(1)_{M}$ has weights $(1,-1,0,0)$ on the coordinates $\left(z_{1}, z_{2}, z_{3}, z_{4}\right)$ on $\mathbb{C}^{4} \supset S^{7}$. This fixes a copy of $\mathscr{F}=S^{3} \subset S^{7}$ at $z_{1}=z_{2}=0$, which then becomes a D6-brane locus in the type IIA spacetime $M_{6}=S^{7} / \mathrm{U}(1)_{M}=S^{6}$. The dual field theory is then the low-energy gauge theory on $N$ D2-branes in flat spacetime, which in $\mathcal{N}=2$ language is a $\mathrm{U}(N)$ gauge theory with adjoint fields $X_{1}, X_{2}, X_{3}$ and cubic superpotential ( $\mathcal{N}=8$ super-Yang-Mills), but with additional fundamental fields arising from the low-energy string modes stretching between the D2-branes and D6-brane. This gives rise to additional fields $q, \tilde{q}$ in the fundamental and anti-fundamental of $\mathrm{U}(N)$ respectively, and a corresponding additional superpotential term (see [13]). This is often called the mirror to the ABJM theory, and indeed both theories have superconformal fixed points that are dual to $\mathrm{AdS}_{4} \times S^{7}$. The gauge theories are of course quite different, one being a $\mathrm{U}(N) \times \mathrm{U}(N)$ gauge theory, the other being a $\mathrm{U}(N)$ gauge theory. ${ }^{3}$

In the above cases the type IIA description is under control and typically wellunderstood, allowing one to determine an appropriate UV gauge theory. We shall see more complicated examples in section 4 .

\subsection{BPS M2-brane probes}

The supersymmetric M2-brane which is conjectured to be holographically dual to the Wilson loop on $S^{3}$ must necessarily have as boundary a Hopf circle in $S^{3}$. A convenient explicit form for the Euclidean $\mathrm{AdS}_{4}$ metric can be taken to be

$$
g_{\mathrm{AdS}_{4}}=\frac{\mathrm{d} q^{2}}{1+q^{2}}+q^{2} \mathrm{~d} \Omega_{3}
$$

with $\mathrm{d} \Omega_{3}$ the round metric on the unit sphere $S^{3}$, and $q \in[0, \infty)$ a radial coordinate. The M2-branes of interest then wrap $\Sigma_{2} \times S_{M}^{1}$, where the surface $\Sigma_{2} \subset \operatorname{AdS}_{4}$ has boundary $\partial \Sigma_{2}=S_{\mathrm{Hopf}}^{1} \subset S^{3}$, and $S_{M}^{1} \subset Y_{7}$ is the M-theory circle. The submanifold $\Sigma_{2}$ is then parametrized by the radial direction $q$ in $\mathrm{AdS}_{4}$, and a geodesic Hopf circle $S_{\mathrm{Hopf}}^{1}$ in $S^{3}$, whilst $S_{M}^{1} \subset Y_{7}$ is a priori arbitrary (imposing supersymmetry will later give restrictions on $\left.S_{M}^{1}\right)$. The area of the surface $\Sigma_{2}$ in $\mathrm{AdS}_{4}$ is divergent, but can be regularized by subtracting the length of its boundary, i.e. the length of the $S_{\text {Hopf }}^{1}$ geodesic in $S^{3}$ at $q \rightarrow \infty$. Notice this is then a local boundary counterterm. Including also the warp factor one finds the regularized area to be

$$
\operatorname{Vol}\left(\Sigma_{2}\right)=-\frac{\pi}{2} \mathrm{e}^{2 \Delta}
$$

\footnotetext{
${ }^{3}$ It happens that the Wilson loops turn out to be the same in these theories (essentially due to the high degree of symmetry), but the spectrum of BPS M2-branes/fundamental strings is certainly different. See section 4.1
} 
The action of the M2-brane then reads

$$
S_{\mathrm{M} 2}=\frac{\operatorname{Vol}\left(\Sigma_{2} \times S_{M}^{1}\right)}{(2 \pi)^{2} \ell_{p}^{3}}=-\frac{1}{(2 \pi)^{2} \ell_{p}^{3}} \frac{\pi}{2} \int_{S_{M}^{1}} \mathrm{e}^{3 \Delta} \operatorname{vol}_{S_{M}^{1}},
$$

where $\operatorname{vol}_{S_{M}^{1}}$ is the volume form on $S_{M}^{1}$ induced from the metric $g_{Y_{7}}$.

As mentioned above, imposing that the M2-brane $\Sigma_{2} \times S_{M}^{1}$ is supersymmetric gives restrictions on the possible circles $S_{M}^{1}$. To see this, we need to split the Clifford algebra Cliff $(11,0)$ generated by gamma matrices $\Gamma_{A}$ satisfying $\left\{\Gamma_{A}, \Gamma_{B}\right\}=2 \delta_{A B}$ into $\operatorname{Cliff}(4,0) \otimes$ $\operatorname{Cliff}(7,0)$ via

$$
\Gamma_{\alpha}=\rho_{\alpha} \otimes 1, \quad \Gamma_{a+3}=\rho_{5} \otimes \gamma_{a},
$$

where $\alpha, \beta=0,1,2,3$ and $a, b=1, \ldots, 7$ are orthonormal frame indices for Euclidean $\mathrm{AdS}_{4}$ and $Y_{7}$ respectively, $\left\{\rho_{\alpha}, \rho_{\beta}\right\}=2 \delta_{\alpha \beta},\left\{\gamma_{a}, \gamma_{b}\right\}=2 \delta_{a b}$ and we have defined $\rho_{5} \equiv \rho_{0} \rho_{1} \rho_{2} \rho_{3}$. If we denote by $X^{M}$ the embedding coordinates of the worldvolume of the M2-brane into the target geometry, the amount of preserved supersymmetry is equal to the number of spinors $\epsilon$, as in (3.2), satisfying the projection condition [53]

$$
\mathbb{P} \epsilon=0, \quad \text { where } \mathbb{P} \equiv \frac{1}{2}\left(1-\frac{\mathrm{i}}{3 !} \varepsilon^{i j k} \partial_{i} X^{M} \partial_{j} X^{N} \partial_{k} X^{P} \Gamma_{M N P}\right),
$$

with $i, j, k$ indices on the worldvolume. We now choose an orthonormal frame in elevendimensions as (cf. (3.1))

$$
E^{0}=\frac{1}{2} \mathrm{e}^{\Delta} \frac{\mathrm{d} q}{\sqrt{1+q^{2}}}, \quad E^{m}=\frac{1}{2} \mathrm{e}^{\Delta} q e^{m}, \quad E^{3+a}=\mathrm{e}^{\Delta} e_{Y_{7}}^{a},
$$

where $\left\{e^{m}\right\}_{m=1,2,3}$ is an orthonormal frame on $S^{3}$ and $\left\{e_{Y_{7}}^{a}\right\}_{a=1, \ldots .7}$ is an orthonormal frame on $\left(Y_{7}, g_{7}\right)$, with $e_{Y_{7}}^{1}$ (or rather its dual vector field) aligned along the M-theory circle vector field $\zeta_{M}$. Taking $e^{3}$ to be aligned along the Hopf circle, as in section 2.1, the projector $\mathbb{P}$ then takes the simple form

$$
\mathbb{P}=\frac{1}{2}\left(1-\mathrm{i} \rho_{5} \rho_{03} \otimes \gamma_{1}\right)
$$

and the constraints that follow on the spinors $\psi_{ \pm}, \chi_{ \pm}$on Euclidean $\mathrm{AdS}_{4}$ and $Y_{7}$, respectively, are

$$
\left(1-\mathrm{i} \rho_{5} \rho_{03}\right) \psi_{ \pm}=0, \text { and }\left(1-\gamma_{1}\right) \chi_{ \pm}=0 .
$$

In order to determine how much supersymmetry is preserved by the brane in $\mathrm{AdS}_{4}$, we must count the number of Killing spinors $\psi_{ \pm}$that satisfy the last projection equation. We may decompose the four-dimensional gamma matrices into $\rho_{0}=1 \otimes \tau_{3}$ and $\rho_{\mu}=\tau_{\mu} \otimes \tau_{1}$, with the Pauli matrices $\tau_{\mu}, \mu=1,2,3$. These matrices act on spinors of the form $\psi=\left(\psi_{1}, \psi_{2}\right)^{T}$, with $\psi_{1,2} 2$-component spinors. The Killing spinors on $\mathrm{AdS}_{4}$ may then be constructed from Killing spinors on the $S^{3}$ at fixed radial coordinate $q$. Explicitly, if $\varepsilon$ solves the Killing spinor equation

$$
\nabla_{\mu} \varepsilon=\frac{\mathrm{i}}{2} \tau_{\mu} \varepsilon
$$


on $S^{3}$, then

$$
\psi=\left(\begin{array}{c}
\left(q+\sqrt{1+q^{2}}\right)^{1 / 2} \varepsilon \\
\left(q+\sqrt{1+q^{2}}\right)^{-1 / 2} \varepsilon
\end{array}\right),
$$

is a Killing spinor on Euclidean $\mathrm{AdS}_{4}$. Equation (3.17) has two solutions, one being chiral and one anti-chiral, i.e. $\tau_{3} \varepsilon= \pm \varepsilon$. One then easily shows that the first projection equation in (3.16) is satisfied if we restrict to chiral $\varepsilon$ in the last solution, which singles out one of these two spinors on $\mathrm{AdS}_{4} \cdot{ }^{4}$ Hence the M2-brane preserves half of the supersymmetry in $\mathrm{AdS}_{4}$. Note that the same positive chirality condition also appeared in the supersymmetry condition derived in the field theory context, cf. $(2.6) .^{5}$

The second projection equation in (3.16) tells us which circles $S_{M}^{1}$ give rise to supersymmetry-preserving M2-branes. Following a standard argument one notices that

$$
\bar{\chi}_{+}\left(\frac{1-\gamma_{1}}{2}\right) \chi_{+}=\bar{\chi}_{+}\left(\frac{1-\gamma_{1}}{2}\right)^{\dagger}\left(\frac{1-\gamma_{1}}{2}\right) \chi_{+}=\left|\left(\frac{1-\gamma_{1}}{2}\right) \chi_{+}\right|^{2} \geq 0,
$$

using $\gamma_{1}=\gamma_{1}^{\dagger}$ and $\gamma_{1}^{2}=1$. This immediately gives $\operatorname{vol}_{S_{M}^{1}} \geq \bar{\chi}_{+} \gamma_{(1)} \chi_{+}$(with a pull-back understood), with equality if and only if some supersymmetry is preserved by $S_{M}^{1}$. The action (3.11) for a supersymmetric brane is then

$$
S_{\mathrm{M} 2}=\frac{\operatorname{Vol}\left(\Sigma_{2} \times S_{M}^{1}\right)}{(2 \pi)^{2} \ell_{p}^{3}}=-\frac{1}{(2 \pi)^{2} \ell_{p}^{3}} \frac{\pi}{2} \int_{S_{M}^{1}} \mathrm{e}^{3 \Delta} \bar{\chi}_{+} \gamma_{(1)} \chi_{+} .
$$

With the help of equations (3.3) and (3.8) the action of a supersymmetric M2-brane can be rewritten in terms of the contact form $\eta$ as (taking a convention in which $m<0$ )

$$
S_{\mathrm{M} 2}=-\frac{(2 \pi)^{2} \int_{S_{M}^{1}} \eta}{\sqrt{2 \int_{Y_{7}} \eta \wedge(\mathrm{d} \eta)^{3}}} N^{1 / 2} .
$$

\subsection{M-theory hamiltonian function}

In this subsection we further elucidate the geometry associated to these supersymmetric M2-branes. This geometric structure will both be of practical use, when we come to compute the M2-brane actions (3.21) in examples, and also, as we will see, is realized rather directly in the large $N$ dual matrix model.

We begin by introducing the $M$-theory Hamiltonian function

$$
\left.h_{M} \equiv \eta\left(\zeta_{M}\right)=\zeta_{M}\right\lrcorner \eta
$$

where $\zeta_{M}$ generates the M-theory circle action. This is a real function on $Y_{7}$, and since $\zeta_{M}$ is assumed to preserve the Killing spinors and metric on $Y_{7}$, it follows that $\zeta_{M}$ preserves $h_{M}$ and commutes with the Reeb vector field $\xi$. It follows that the contact length of an

\footnotetext{
${ }^{4}$ The other two Killing spinors on $\mathrm{AdS}_{4}$ are constructed from spinors on $S^{3}$ satisfying $\nabla_{\mu} \varepsilon=-\frac{\mathrm{i}}{2} \tau_{\mu} \varepsilon$. We set the corresponding spinors to zero in section 2, as they are not used in the supersymmetric localization. Again, one chirality is broken by the M2-brane.

${ }^{5}$ Notice that the Wilson loop circle $\gamma \subset S^{3}$ is calibrated by $e^{3}$, one of the left-invariant one-forms under $\mathrm{SU}(2)_{\text {left }}$, which is a contact form on $S^{3}$.
} 
M-theory circle $S_{M}^{1}$ over a point $p \in M_{6}=Y_{7} / \mathrm{U}(1)_{M}$ is given by $\int_{S_{M}^{1}} \eta=2 \pi h_{M}(\hat{p})$, where $\hat{p} \in Y_{7}$ is any lift of the point $p$. This directly leads to the form of the M2-brane action (1.4).

One way to characterize the supersymmetric M-theory circles $S_{M}^{1}$ is to note that on $\left.T Y_{7}\right|_{S_{M}^{1}}$ the vector $\zeta_{M}$ is necessarily proportional to the Reeb vector. Indeed, using (3.1) one can show that at these supersymmetric points

$$
\left.\zeta_{M}\right\lrcorner \mathrm{d} \eta=0 .
$$

To see this one takes the projection condition (3.16) with $\chi_{-}$, applies $\bar{\chi}_{+}^{c} \gamma_{a}$ on the left, and then takes the real part of the resulting equation. Using $\operatorname{Re}\left[\bar{\chi}_{+}^{c} \chi_{-}\right]=\operatorname{Re}\left[\bar{\chi}_{+}^{c} \gamma_{a} \chi_{-}\right]=0$ and the relation between $\mathrm{d} \eta$ and $\operatorname{Re}\left[\bar{\chi}_{+}^{c} \gamma_{(2)} \chi_{-}\right]$in (3.1) then leads to (3.23). That this then implies $\zeta_{M} \propto \xi$ follows from the fact that $\eta$ is a contact form: $\mathrm{d} \eta$ is a symplectic form on ker $\eta$, the rank 6 subbundle of the tangent bundle $T Y_{7}$ of $Y_{7}$ defined as vectors having zero contraction with $\eta$. Since this means that $\mathrm{d} \eta$ is non-degenerate on this rank 6 bundle, and since also $T Y_{7}=\operatorname{ker} \eta \oplus\langle\xi\rangle$, where $\langle\xi\rangle$ is the real line bundle spanned by vectors proportional to $\xi$, (3.23) implies that the projection of $\zeta_{M}$ onto $\operatorname{ker} \eta$ is zero, i.e. that $\zeta_{M} \propto \xi$.

The condition (3.23) is then also the condition that we are at a critical point of the Hamiltonian $h_{M}$. To see this, we rewrite $\mathcal{L}_{\zeta_{M}} \eta=0$ using the Cartan formula, so that (3.23) is equivalent to

$$
\left.\mathrm{d}\left(\zeta_{M}\right\lrcorner \eta\right)=0 \quad \Leftrightarrow \quad \mathrm{d} h_{M}=0 .
$$

Thus the supersymmetric M2-branes lie precisely on the critical set $\left\{\mathrm{d} h_{M}=0\right\}$, and their action (1.4) is determined by $h_{M}$ evaluated at the critical point! It is a general fact that any component of the moment map for a compact group action on a symplectic manifold is a Morse-Bott function. Here more precisely recall that the cone $C(Y)=\mathbb{R}_{\geq 0} \times Y_{7}$ is symplectic, with symplectic form

$$
\omega=\frac{1}{2} \mathrm{~d}\left(r^{2} \eta\right)
$$

where $r \geq 0$ is a radial coordinate. In fact the cone being symplectic is equivalent to $\left(Y_{7}, \eta\right)$ being contact. The M-theory circle action then gives a $\mathrm{U}(1)_{M}$ action on this cone, with moment map

$$
\left.\mu=\frac{1}{2} r^{2} \zeta_{M}\right\lrcorner \eta
$$

Thus $\mu$ is Morse-Bott, and the restriction of $\mu$ to $Y_{7}$ at $r=1$ is our Hamiltonian function $h_{M} / 2$. We thus know that the image $h_{M}\left(Y_{7}\right)=\left[c_{\min }, c_{\text {max }}\right]$ is a closed interval, and this is further subdivided into $P$ intervals via $c_{\min }=c_{1}<c_{2}<\cdots<c_{P+1}=c_{\max }$, where the $c_{i}$ are images under $h_{M}$ of the critical set $\left\{\mathrm{d} h_{M}=0\right\}$. On each open interval $c \in\left(c_{i}, c_{i+1}\right)$ the level surfaces $h_{M}^{-1}(c)$ are all diffeomorphic to the same fixed six-manifold, with the topology changing as one crosses a critical point $c_{i}$.

Finally, since at a supersymmetric $S_{M}^{1}$ we have $\zeta_{M} \propto \xi$, it follows that the corresponding point $p \in M_{6}=Y_{7} / \mathrm{U}(1)_{M}$ is a fixed point under the induced Reeb vector action on 
$M_{6}=Y_{7} / \mathrm{U}(1)_{M}$. That is, over every fixed point $p \in M_{6}$ of $\xi$, there exists a calibrated and supersymmetric M-theory circle $S_{M, p}^{1}$ whose corresponding supersymmetric M2-brane action is given by (1.4).

In the holographic computation of the Wilson loop VEV via the M2-brane action, one should sum $\mathrm{e}^{-S_{\mathrm{M} 2, p}}$ over all contributions. In some cases (typically with more symmetry) we shall find that the supersymmetric points $p \in M_{6}$ form submanifolds which are fixed by $\xi$, and this sum in fact becomes an integral over the different connected submanifolds. Notice that $h_{M}$ is constant on each connected component of the fixed point set. In any case, in the large $N$ limit only the longest circle $S_{M}^{1}$ survives, the others being exponentially suppressed relative to it in the sum/integral, hence proving formula (1.1).

The calculation of the action of a supersymmetric M2-brane can be completely carried out once the Reeb vector field $\xi$ and the M-theory circle generator $\zeta_{M}$ are known. Indeed, the contact volume $\operatorname{Vol}_{\eta}\left(Y_{7}\right)$ is a function only of the Reeb vector [45], and the length of a

calibrated circle $\int_{S_{M, p}^{1}} \eta=2 \pi h_{M}(\hat{p})$ depends only on $\xi, \zeta_{M}$ and the point $p$. Even though this could appear to be involved, the computation of these two quantities is relatively straightforward for appropriate classes of $Y_{7}$. In particular, if we focus on toric manifolds, standard geometrical techniques can be exploited to straightforwardly find all calibrated circles, i.e. the connected components of the critical set $\left\{\mathrm{d} h_{M}=0\right\} \subset Y_{7}$, as well as the contact volume [49]. This is the subject of the next subsection.

\subsection{Geometric methods of computation}

In this section we explain how to compute the various quantities we have been discussing in appropriate classes of examples. We focus our discussion on toric geometries, which means that $\mathrm{U}(1)^{4}$ acts on $Y_{7}$, preserving the contact form $\eta$. In this case there are some pretty geometric methods, first developed in [48, 49], that may be utilized to calculate the length of the calibrated M-theory circles, as well as the volumes of the internal spaces. We will thus focus on this class of solutions, although we note that the more general methods described in [49] may be used to attack non-toric cases.

Let us begin with the symplectic cone $\left(C(Y)=\mathbb{R}_{\geq 0} \times Y, \omega=\frac{1}{2} \mathrm{~d}\left(r^{2} \eta\right)\right)$, but in general dimension $2 n$. Equivalently, $(Y, \eta)$ is contact with $\operatorname{dim} Y=2 n-1$. The toric condition means that $\mathrm{U}(1)^{n}$ acts on the symplectic cone $C(Y)$ preserving the symplectic form $\omega$, and we may parametrize the generating vector fields as $\partial_{\phi_{i}}$, with $\phi_{i} \in[0,2 \pi)$ and $i=1, \ldots, n$. This allows one to introduce symplectic coordinates $\left(y_{i}, \phi_{i}\right)$ in which the symplectic form on $C(Y)$ has the simple expression

$$
\omega=\sum_{i=1}^{n} \mathrm{~d} y_{i} \wedge \mathrm{d} \phi_{i} .
$$

Moreover, when the toric cone is of Reeb type, meaning that $\xi$ is in the Lie algebra of $\mathrm{U}(1)^{n}$, the coordinates $y_{i}$ take values in a convex polyhedral cone $\mathcal{C}^{*} \subset \mathbb{R}^{n}$ [54]. If this cone has $d$ facets, we have corresponding outward primitive normal vectors to these facets, $v_{a} \in \mathbb{Z}^{n}$, $a=1, \ldots, d$, with the facets corresponding (under the moment map) to the fixed point sets of $\mathrm{U}(1) \subset \mathrm{U}(1)^{n}$ with weights $v_{a}$. In particular this set-up applies to toric Sasakian $Y$ [48], 
in which the symplectic cone $C(Y)$ is also Kähler. In this case, the topological condition that $C(Y)$ is Calabi-Yau (more precisely, that the apex $\{r=0\}$ is a Gorenstein singularity) is equivalent to the existence of an $\mathrm{SL}(n, \mathbb{Z})$ transformation such that the normal vectors take the form $v_{a}=\left(1, w_{a}\right)$, for all $a$, with $w_{a} \in \mathbb{Z}^{n-1}$. In this basis, the first component of the Reeb vector is necessarily $\xi_{1}=n$ [48].

In general the components of $\xi=\sum_{i=1}^{n} \xi_{i} \partial_{\phi_{i}}$ form a vector $\vec{\xi}=\left(\xi_{1}, \ldots, \xi_{n}\right)$ that defines the characteristic hyperplane in $\mathbb{R}^{n}:\left\{\vec{y} \in \mathbb{R}^{n} \mid \vec{\xi} \cdot \vec{y}=\frac{1}{2}\right\}$. This hyperplane interesects $\mathcal{C}^{*}$ to form a finite polytope $\Delta_{\xi}$, and the contact volume of the base $Y$ is related to the volume of this polytope by ${ }^{6}$

$$
\operatorname{Vol}_{\eta}(Y)=2 n(2 \pi)^{n} \operatorname{Vol}\left(\Delta_{\xi}\right) .
$$

Moreover, each of the $d$ facets $\mathcal{F}_{a}$, intersected with the characteristic hyperplane, are images under the moment map of $(2 n-3)$-dimensional subspaces $\Sigma_{a}$ of $Y$. The volumes of these submanifolds may be calculated once the volumes of the facets are known, for

$$
\operatorname{Vol}_{\eta}\left(\Sigma_{a}\right)=(2 n-2)(2 \pi)^{n-1} \frac{1}{\left|v_{a}\right|} \operatorname{Vol}\left(\mathcal{F}_{a}\right)
$$

In addition, the volume of the base manifold $Y$ is simply given by

$$
\operatorname{Vol}_{\eta}(Y)=\frac{(2 \pi)^{n}}{\xi_{1}} \sum_{a=1}^{d} \frac{1}{\left|v_{a}\right|} \operatorname{Vol}\left(\mathcal{F}_{a}\right) .
$$

In [49] the idea is to study the space of Kähler cone metrics on $C(Y)$, and thus Sasakian structures on $Y$. One then considers the Einstein-Hilbert action (with a fixed positive cosmological constant) restricted to this space of Sasakian metrics on $Y$, so that a SasakiEinstein metric on $Y$ is a critical point. In fact the action is minimized and proportional to the volume of the base $\operatorname{Vol}(Y)$ when the metric on $Y$ is Sasaki-Einstein. In this case there is unique Reeb vector of the form $\vec{\xi}=\left(n, \xi_{2}, \ldots, \xi_{n}\right)$ such that the Einstein-Hilbert action, or equivalently $\operatorname{Vol}(Y)$, is minimized as a function of $\xi$. Thus, for any given toric diagram one calculates $\operatorname{Vol}(Y)$ with formula (3.30) as a function of the Reeb vector, and determines $\vec{\xi}$ for the Sasaki-Einstein metric on $Y$ by minimizing this function. ${ }^{7}$ Presumably these ideas extend to more general warped geometries, with non-zero internal flux $F_{4} \neq 0$ in (3.1), following a similar construction in type IIB $\mathrm{AdS}_{5}$ solutions [45].

In this paper we need only apply this method for $n=4$. A way to compute $\operatorname{Vol}\left(\mathcal{F}_{a}\right)$ as a function of the Reeb vector for $n=4$ has been described in [8]. If the facet $\mathcal{F}_{a}$ is a tetrahedron, its vertex is at the origin in $\mathcal{C}^{*}$ and its base is a triangle lying in the characteristic hyperplane. This is generated by three edges passing from the characteristic hyperplane to the origin, and bounded by four hyperplanes creating the polyhedron. In addition to $v_{a}$, three other facets are then involved in the construction of the tetrahedron, and we denote their normal vectors as $v_{a, 1}, v_{a, 2}, v_{a, 3}$. The volume of the tetrahedron may be expressed as

$$
\frac{1}{\left|v_{a}\right|} \operatorname{Vol}\left(\mathcal{F}_{a}\right)=\frac{1}{48} \frac{\left(v_{a}, v_{a, 1}, v_{a, 2}, v_{a, 3}\right)^{2}}{\left|\left(\xi, v_{a}, v_{a, 1}, v_{a, 2}\right)\left(\xi, v_{a}, v_{a, 1}, v_{a, 3}\right)\left(\xi, v_{a}, v_{a, 2}, v_{a, 3}\right)\right|},
$$

\footnotetext{
${ }^{6}$ In the Sasakian case the Riemannian volume and contact volumes coincide.

${ }^{7}$ That the Sasaki-Einstein metric indeed always exists was proven in [55].
} 
with $(\cdot, \cdot, \cdot, \cdot)$ the determinant of a $4 \times 4$ matrix. If the facet $\mathcal{F}_{a}$ is not a tetrahedron, i.e. there are more than 3 edges that meet at a vertex in the toric diagram (cf. below), the volume can be computed with the same formula by breaking up the facet into tetrahedrons.

The toric diagram for a toric Calabi-Yau cone is by definition the convex hull of the lattice vectors $w_{a}$ in $n-1=3$ dimensions. To each vertex in this diagram corresponds a facet $\mathcal{F}_{a}$. If the vertex is located at the intersection of three planes, or equivalently three edges of the toric diagram meet at the vertex, then it corresponds to a tetrahedron. If instead four edges meet at the vertex, the facet is a pyramid that can be split into two tetrahedrons, and so on. A given facet $\mathcal{F}_{a}$ then corresponds to a vector $v_{a}=\left(1, w_{a}\right)$, with $w_{a}$ a vertex in the toric diagram; the other three vectors $v_{a, 1}, v_{a, 2}, v_{a, 3}$ are the outwardpointing primitive vectors corresponding in the toric diagram to the three edges that meet at the vertex $v_{a}$. Let us also note that the base $Y_{7}$ of the cone is a smooth manifold only if each face of the toric diagram is a triangle, and there are no lattice points internal to any edge or face. These conditions are equivalent [56] to the cone being good, in the sense of [54].

It should now be clear that once a toric diagram is given for a toric Calabi-Yau cone

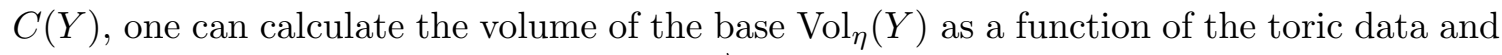
the Reeb vector that is parametrized by $\vec{\xi}=\left(4, \xi_{2}, \xi_{3}, \xi_{4}\right)$. After minimizing the volume with respect to $\xi_{2}, \xi_{3}, \xi_{4}$, one obtains the Reeb vector and $\operatorname{Vol}_{\eta}(Y)$ as a function of the toric data only. For more general warped solutions with flux the cone is not Ricci-flat Kähler, but in the examples we shall study later in section 4 the Reeb vector $\vec{\xi}$ and toric contact structure are in fact known [25].

Next we turn to the M-theory Hamiltonian function $h_{M}$, and the computation of the calibrated circles in $Y_{7}$ and their lengths. This involves, by definition, the choice of an M-theory circle $\zeta_{M}$, as described in section 3.2. As we proved in the last section, supersymmetric calibrated $S_{M}^{1}$ exist where $\zeta_{M}$ is parallel to $\xi$. This is equivalent to

$$
\zeta_{M}=\eta\left(\zeta_{M}\right) \xi=h_{M} \xi
$$

as follows by taking the contraction of each side with $\eta$. We can conclude that if we know the proportionality constant between $\zeta_{M}$ and $\xi$, the length of the corresponding calibrated M-theory circle, located over a fixed point $p$ under $\xi$ in $M_{6}$, is then simply $2 \pi h_{M}(\hat{p})$ with $\hat{p} \in Y_{7}$ any point projecting to $p$. In terms of the toric geometry above, notice that

$$
h_{M}=2 \sum_{i=1}^{n} y_{i} \zeta_{M}^{i},
$$

where $\zeta_{M}=\sum_{i=1}^{n} \zeta_{M}^{i} \partial_{\phi_{i}}$. This may be regarded as a function on the polytope $\Delta_{\xi}$, that is the image of $Y_{7}$ under the moment map.

The only remaining question is how to find where the two vectors $\zeta_{M}, \xi$ are proportional to each other, or equivalently what are the critical points of $h_{M}$, and also what is the value of $h_{M}$ at those points. With the formalism at hand, this is straightforward to answer. Once a toric diagram and $\zeta_{M}$ are given, the Reeb vector (and the volume) can be found 
with the method described above. We may then find the solutions to the equation

$$
\zeta_{M}=\beta \xi+\sum_{a \in I} \alpha_{a} v_{a}
$$

with $\beta, \alpha_{a}$ real numbers, and $I \subset\{1, \ldots, d\}$ a subset of three facets which intersect. Geometrically, the intersection of three facets defines an edge of $\mathcal{C}^{*}$, which corresponds to a circle $S^{1} \subset Y_{7}$. This circle is a fixed point set of $\mathrm{U}(1)^{3} \subset \mathrm{U}(1)^{4}$ defined by the three vectors $v_{a}, a \in I$, meaning that the generating $\mathrm{U}(1)$ vector fields corresponding to $v_{a}$ are zero over this circle, and hence $\zeta_{M}$ is parallel to $\xi$. Thus this $S^{1}$ is precisely a calibrated circle. The proportionality constant is then $h_{M}=\eta\left(\zeta_{M}\right)=\beta$, and its length is $2 \pi h_{M}$. Thus our problem boils down to linear algebra on the polyhedral cone.

We make a few further geometrical observations. First, if (3.34) holds with $\beta=0$ then $\zeta_{M}$ actually fixes the $S^{1}$, meaning that there must be D6-branes present. The M-theory circle then has zero length on such loci, formally leading to M2-branes with zero action; if $\zeta_{M}$ acts freely on $Y_{7}$ this cannot happen. Next we note that (3.34) cannot hold with $\alpha_{a}=0$ for all $a \in I$, since then $\zeta_{M}$ would be everywhere parallel to $\xi$, and this cannot happen since $\zeta_{M}$ is a non-R symmetry. However, it may happen that (3.34) holds with one or two (but not all three) of the coefficients $\alpha_{a}=0$. Geometrically, this means that in this case $\zeta_{M}$ is parallel to $\xi$ over the intersection of (respectively) the corresponding two or one facets with non-zero $\alpha_{a}$ coefficients, leading to three-dimensional or five-dimensional subspaces of $Y_{7}$ which are fibred by calibrated $S_{M}^{1}$ circles. These then descend to two-dimensional or four-dimensional fixed point sets of $\xi$ on $M_{6}=Y_{7} / \mathrm{U}(1)_{M}$, respectively. We shall see examples of this in the next section. Finally, if the toric diagram contains faces which have more than three sides, then (3.34) may hold for $I$ being the corresponding set of 4 or more vectors $v_{a}$. In this case the manifold has a locus of (worse than orbifold) singularities along the corresponding $S^{1}$ in $Y_{7}$, and our theory above doesn't directly apply to these singular circles (their tangent spaces are not even a quotient of $\mathbb{R}^{7}$ ).

Even though the above theoretical background may appear cumbersome, it is effectively not difficult to find the volume of $Y_{7}$, its Reeb vector $\xi$ and all the calibrated circles and their lengths. Thanks to equation (1.4), the action for each corresponding M2-brane follows straightforwardly, and can be compared to the data extracted from the matrix model of the dual field theory. We examine these computations, in a variety of examples, in the next section.

\subsection{Hamiltonian function and density}

In $[61,62]$ a relation was also found between $\rho(x)$, and other matrix model variables, and certain geometric invariants. In particular, equation (1.4a) of [62] relates $\rho(x)$ to the derivative of a function that counts operators in the chiral ring of the gauge theory according to their R-charge and monopole charges. In the language of the current paper, the monopole charge is the charge under $\mathrm{U}(1)_{M}$. With our notations and conventions, 
using [62] one can rewrite their conjecture for $\rho(x)$ in the following form:

$$
\begin{gathered}
\rho(x)=\left.\frac{4}{\pi^{2}} \frac{(2 \pi)^{3}}{\sqrt{96 \operatorname{Vol}_{\eta}\left(Y_{7}\right)}} \frac{\partial_{r} \operatorname{vol}\left(P_{r c}\right)}{\left|\xi \wedge \zeta_{M}\right|}\right|_{r=1}, \\
\text { where } \quad P_{r c} \equiv\left\{y \in \mathcal{C}^{*} \mid \vec{y} \cdot \vec{\xi}=\frac{r}{2}, \vec{y} \cdot \vec{\zeta}_{M}=\frac{c}{2}\right\},
\end{gathered}
$$

where the variable $c$ is related to $x$ by (1.6). Using equation (3.33), we know that for the toric case $\vec{y} \cdot \vec{\zeta}_{M}=\frac{1}{2} h_{M}$. If we introduce

$$
P_{c} \equiv\left\{y \in \mathcal{C}^{*} \mid h_{M}=c\right\},
$$

we see that $P_{r c}$ is nothing but the intersection of $P_{c}$ with the characteristic hyperplane. But since the pre-image (under the moment map) of $P_{c}$ in $Y_{7}$ is the same as $h_{M}^{-1}(c)$, which changes topology every time we pass through a critical point of $h_{M}$, we know that the topology of the pre-image of $P_{r c}$ in $Y_{7}$ also changes every time a critical point is crossed. Thus we expect a change of behaviour of $\operatorname{vol}\left(P_{r_{c}}\right)$ and hence $\rho(x)$ at the critical points $x_{i}$ that are related to the $c_{i}$ by (1.6). In other words, the eigenvalue density has a different behaviour in each subset $\left(c_{i}, c_{i+1}\right)$, as we will see in the examples in the next section, because there are supersymmetric M2 branes located at the $c_{i}$, which are critical points of a Hamiltonian function. That explains why the function $\rho(x)$ has a jump in its derivative precisely at the critical points.

\section{Examples}

In this section we illustrate the duality between geometries and matrix models in a wide variety of examples. In particular we will compute the image of the M-theory Hamiltonian $h_{M}\left(Y_{7}\right)=\left[c_{\min }, c_{\max }\right]$, and show that it coincides with the support of the matrix model eigenvalues $\left[x_{\min }, x_{\max }\right]$ via (1.6). The critical points of $h_{M}$ will be shown to map to the points $x=x_{i}$ where $\rho^{\prime}(x)$ has a jump discontinuity, with the matching of Wilson loops being a corollary of this result for $x=x_{\max }$.

\subsection{Duals to the round $S^{7}$}

We begin by studying two superconformal duals to $\mathrm{AdS}_{4} \times S^{7}$, where $S^{7}$ is equipped with its standard round Einstein metric. These differ in the choice of M-theory circle $\mathrm{U}(1)_{M}$ acting on $S^{7}$, as we discussed briefly in section 3.2. In this case the geometry is particularly simple, allowing us to illustrate the geometric structures we have described very explicitly.

\subsubsection{ABJM theory}

The ABJM theory [1] is an $\mathcal{N}=6$ superconformal $\mathrm{U}(N)_{k} \times \mathrm{U}(N)_{-k}$ Chern-Simons-matter theory. In $\mathcal{N}=2$ language, there are two chiral matter fields $A_{1}, A_{2}$ in the bifundamental $(\mathbf{N}, \overline{\mathbf{N}})$ representation of this gauge group, two chiral matter fields $B_{1}, B_{2}$ in the conjugate $(\overline{\mathbf{N}}, \mathbf{N})$ representation, and a quartic superpotential. Here the subscript $k \in \mathbb{Z}$ in $\mathrm{U}(N)_{k}$ 
denotes the Chern-Simons level for the particular copy of $\mathrm{U}(N)$, as in (2.2). This theory is dual to $\mathrm{AdS}_{4} \times S^{7} / \mathbb{Z}_{k}$ with $N$ units of flux (3.8), where $\mathbb{Z}_{k} \subset \mathrm{U}(1)_{\text {Hopf }}=\mathrm{U}(1)_{M}$.

We may realize $S^{7}$ as the unit sphere $S^{7} \subset \mathbb{R}^{8} \cong \mathbb{C}^{4}$ and take $\mathrm{U}(1)_{\text {Hopf }}$ to have weights $(1,1,-1,-1)$ on the four complex coordinates $\left(z_{1}, z_{2}, z_{3}, z_{4}\right)$ on $\mathbb{C}^{4}$. In this description the $\mathrm{U}(1)_{R}$ symmetry of the $\mathcal{N}=2$ subalgebra of the $\mathcal{N}=6$ manifest superconformal symmetry of the theory has weights $(1,1,1,1)$ on $\mathbb{C}^{4}$, which gives a different Hopf action on $\mathbb{C}^{4}$. In [1] the variables $C_{\alpha} \equiv\left(A_{1}, A_{2}, B_{1}^{*}, B_{2}^{*}\right)$ were also used. In this choice of complex structure on $\mathbb{R}^{8} \cong \mathbb{C}^{4}$ the $\mathrm{U}(1)_{M}$ and $\mathrm{U}(1)_{R}$ weights above are interchanged; in these variables the $\mathrm{SU}(4)_{R}$ symmetry of the theory, which acts isometrically on $\mathbb{C P}^{3}=\left\{S^{7} \subset \mathbb{C}^{4}\right\} / \mathrm{U}(1)_{M}$, is manifest. However, to be uniform with the other examples we shall study, we shall fix the first complex structure on $\mathbb{R}^{8} \cong \mathbb{C}^{4}$ above.

In these coordinates $S^{7}=\left\{\left.\left(z_{1}, z_{2}, z_{3}, z_{4}\right) \in \mathbb{C}^{4}|| z_{1}\right|^{2}+\left|z_{2}\right|^{2}+\left|z_{3}\right|^{2}+\left|z_{4}\right|^{2}=1\right\}$, while the M-theory Hamiltonian function on $S^{7} / \mathbb{Z}_{k}$ is

$$
h_{M}=\frac{1}{k}\left(\left|z_{1}\right|^{2}+\left|z_{2}\right|^{2}-\left|z_{3}\right|^{2}-\left|z_{4}\right|^{2}\right) .
$$

In the toric geometry language of section 3.5, we have the symplectic coordinates $y_{i}=$ $\frac{1}{2}\left|z_{i}\right|^{2}$. The level sets $h_{M}^{-1}(c)$ are diffeomorphic to $S^{3} \times S^{3} / \mathbb{Z}_{k}$ for $c \in\left(-\frac{1}{k}, \frac{1}{k}\right)$. Perhaps the easiest way to explain this is to note that dividing the levels sets also by $\mathrm{U}(1)_{M}$ gives the Kähler quotient description of $T^{1,1} .8$ The level sets are then a circle bundle over $T^{1,1} \cong S^{2} \times S^{3}$, with first Chern class $k \in \mathbb{Z} \cong H^{2}\left(S^{2} \times S^{3}, \mathbb{Z}\right)$, which means they are diffeomorphic to $S^{3} \times S^{3} / \mathbb{Z}_{k}$. Notice that these level sets are also described by

$$
\left|z_{1}\right|^{2}+\left|z_{2}\right|^{2}=\frac{1}{2}(1+c k), \quad\left|z_{3}\right|^{2}+\left|z_{4}\right|^{2}=\frac{1}{2}(1-c k) .
$$

When $c \rightarrow \pm \frac{1}{k}$ the $S^{3} \times S^{3} / \mathbb{Z}_{k}$ level sets thus collapse to two copies of $S^{3} / \mathbb{Z}_{k}$ at $\left\{z_{3}=z_{4}=\right.$ $0\}$ and $\left\{z_{1}=z_{2}=0\right\}$, respectively. Thus the image $h_{M}\left(S^{7}\right)=\left[-\frac{1}{k}, \frac{1}{k}\right]$, with the endpoints $c_{\max }=-c_{\min }=\frac{1}{k}$ being the only two critical points of the Morse-Bott function $h_{M}$.

The contact form in these coordinates is

$$
\eta=\frac{\mathrm{i}}{2 r^{2}} \sum_{i=1}^{4}\left(z_{i} \mathrm{~d} \bar{z}_{i}-\bar{z}_{i} \mathrm{~d} z_{i}\right), \quad r^{2} \equiv \sum_{i=1}^{4}\left|z_{i}\right|^{2} .
$$

Being Einstein, the contact volume of $S^{7} / \mathbb{Z}_{k}$ is equal to the Riemannian volume, with

$$
\operatorname{Vol}\left(S^{7} / \mathbb{Z}_{k}\right)=\frac{\pi^{4}}{3 k}
$$

Our general formula (1.6) thus implies that the matrix model variable $x$ should be related to the geometric quantity $c$ above via

$$
x=\frac{(2 \pi)^{3}}{\sqrt{96 \operatorname{Vol}\left(S^{7} / \mathbb{Z}_{k}\right)}} c=\pi \sqrt{2 k} c .
$$

\footnotetext{
${ }^{8}$ Of course this is directly related to the construction of the ABJM theory itself, as the M-theory lift of the theory on $N$ D2-branes at the conifold singularity $C\left(T^{1,1}\right)$, with $k$ units of RR two-form flux through the vanishing $S^{2}$.
} 


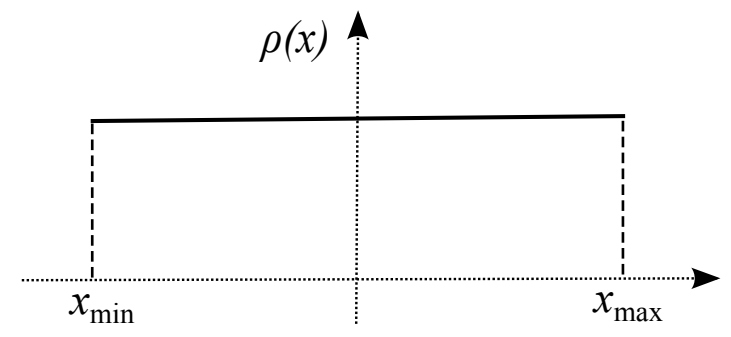

Figure 2. Eigenvalue density $\rho(x)$ for the ABJM theory.

The large $N$ saddle point eigenvalue distribution for the ABJM theory was given in [30]. The eigenvalues for the two gauge groups are related by

$$
\lambda^{1}(x)=\bar{\lambda}^{2}(x)=x N^{1 / 2}+\mathrm{i} y(x),
$$

where

$$
\rho(x)=\frac{\sqrt{k}}{2 \pi \sqrt{2}}, \quad y(x)=\frac{\sqrt{k}}{2 \sqrt{2}} x,
$$

and the eigenvalues are supported on $\left[x_{\min }, x_{\max }\right]$, where $x_{\max }=-x_{\min }=\pi \sqrt{2 / k}$. This of course agrees with the geometric formula (4.5), and since the density $\rho(x)$ is constant on $\left(x_{\min }, x_{\max }\right)$ (see figure 2$)$ its derivative is in particular continuous on this region. It is then automatic that the gravity formula (1.1) agrees with the field theory formula (1.5) for the Wilson loop, giving in both cases

$$
\log \langle W\rangle=\pi \sqrt{\frac{2}{k}} N^{1 / 2}
$$

\subsubsection{Mirror theory}

As discussed in section 3.2, the mirror to the ABJM theory (with $k=1$ ) arises by choosing a different M-theory circle action on $S^{7}$. The field theory [13] is $\mathcal{N}=8 \mathrm{U}(N)$ super-YangMills theory coupled to two additional fields $q, \tilde{q}$ in the fundamental and anti-fundamental representation of $\mathrm{U}(N)$, respectively. The superpotential is

$$
\mathcal{W}=\operatorname{Tr}\left(q X_{1} \tilde{q}+X_{3}\left[X_{1}, X_{2}\right]\right)
$$

where $X_{1}, X_{2}, X_{3}$ are the adjoint chiral fields of the $\mathcal{N}=8$ theory, in $\mathcal{N}=2$ language.

In this case the M-theory circle $\mathrm{U}(1)_{M}$ has weights $(1,-1,0,0)$ on $S^{7} \subset \mathbb{C}^{4}$, which has a codimension four fixed point set $\mathscr{F}=S^{3}=\left\{z_{1}=z_{2}=0\right\} \subset S^{7}$. It follows that the type IIA internal space is $M_{6}=S^{6}$, with a space-filling D6-brane wrapping a copy of $S^{3} \subset S^{6}$. The field theory described in the previous paragraph is then the theory on $N$ D2-branes in flat space ( $\mathcal{N}=8$ super-Yang-Mills), but coupled to additional massless fields $q, \tilde{q}$ arising from the lowest excitations of strings stretching between the D2-branes and D6-brane. 
Although the background geometry is the same as in the previous subsection, the M-theory Hamiltonian is now ${ }^{9}$

$$
h_{M}=\left|z_{1}\right|^{2}-\left|z_{2}\right|^{2} .
$$

The level surfaces $h_{M}^{-1}(c)$ are described by

$$
2\left|z_{1}\right|^{2}+\left|z_{3}\right|^{2}+\left|z_{4}\right|^{2}=1+c, \quad 2\left|z_{2}\right|^{2}+\left|z_{3}\right|^{2}+\left|z_{4}\right|^{2}=1-c,
$$

so that $c \in[-1,1]$. However, the critical point set of $h_{M}$ is quite different to that for the ABJM model (4.1). The endpoints $c=+1, c=-1$ are now the copies of $S^{1} \subset S^{7}$ at $\left\{z_{2}=z_{3}=z_{4}=0\right\}$ and $\left\{z_{1}=z_{3}=z_{4}=0\right\}$, respectively. (Compare to the ABJM model, where for $k=1$ also $c \in[-1,1]$, but with the endpoints being images of copies of $S^{3}$, rather than $S^{1}$.) Moreover, there is an additional critical point at $c=0$, which then intersects the D6-brane locus/fixed point set at $\left\{z_{1}=z_{2}=0\right\}$. Indeed, on $S^{7}$ we have

$$
\begin{aligned}
\mathrm{d} h_{M} & =\left(z_{1} \mathrm{~d} \bar{z}_{1}+\bar{z}_{1} \mathrm{~d} z_{1}\right)-\left(z_{2} \mathrm{~d} \bar{z}_{2}+\bar{z}_{2} \mathrm{~d} z_{2}\right), \\
0 & =\sum_{i=1}^{4}\left(z_{i} \mathrm{~d} \bar{z}_{i}+\bar{z}_{i} \mathrm{~d} z_{i}\right) \quad \Leftrightarrow \quad 0=\mathrm{d} r .
\end{aligned}
$$

Thus in addition to the endpoints $\left\{z_{2}=z_{3}=z_{4}=0\right\}$ and $\left\{z_{1}=z_{3}=z_{4}=0\right\}$, we also have $\mathrm{d} h_{M}=0$ at $\left\{z_{1}=z_{2}=0\right\}=S^{3}$, which is the fixed point set of $\mathrm{U}(1)_{M}$ where $h_{M}=0$. Thus we have the three critical points $c_{1}=c_{\min }=-1, c_{2}=0, c_{3}=c_{\max }=1$.

The topology of the level sets $h_{M}^{-1}(c)$ is the same for $c \in(-1,0)$ and $c \in(0,1)$, but with different circles collapsing on each side. For $c \in(0,1)$ we may "solve" $h_{M}=c$ as $\left|z_{1}\right|^{2}=\left|z_{2}\right|^{2}+c>0$, and note that consequently $z_{1} \neq 0$ on this locus. From (4.11) it follows that $h_{M}^{-1}(c) \cong S_{1}^{1} \times S^{5}$, where $S_{1}^{1}$ is parametrized by the phase of $z_{1}=\left|z_{1}\right| \mathrm{e}^{\mathrm{i} \phi_{1}}$. On the other hand, for $c \in(-1,0)$ instead we solve $h_{M}=c$ as $\left|z_{2}\right|^{2}=\left|z_{1}\right|^{2}-c>0$, so that $h_{M}^{-1}(c) \cong S_{2}^{1} \times S^{5}$, where $S_{2}^{1}$ is parametrized by the phase of $z_{2}=\left|z_{2}\right| \mathrm{e}^{\mathrm{i} \phi_{2}}$.

The general formula (1.6) implies that the matrix model variable $x$ should be related to the geometric quantity $c$ again via

$$
x=\frac{(2 \pi)^{3}}{\sqrt{96 \operatorname{Vol}\left(S^{7}\right)}} c=\pi \sqrt{2} c,
$$

which is the same formula as for the ABJM model with $k=1$. The large $N$ saddle point eigenvalue distribution is in fact a special case of the models in section 4.3, with $a=1$, $b=0$ in the notation of that section, and appears in [33]. In this case there is only a single gauge group, and one finds the eigenvalue density

$$
\rho(x)=\left\{\begin{array}{ll}
\frac{1}{2 \pi^{2}}\left(x-x_{\min }\right), & x_{\min }<x<0 \\
\frac{1}{2 \pi^{2}}\left(x_{\max }-x\right), & 0<x<x_{\max }
\end{array},\right.
$$

where $x_{\max }=-x_{\min }=\pi \sqrt{2}$, thus agreeing with (4.13). Moreover, the derivative of $\rho$ is

\footnotetext{
${ }^{9}$ We could similarly choose to quotient by $\mathbb{Z}_{k} \subset \mathrm{U}(1)_{M}$, which would lead to $k$ D6-branes in the type IIA description. However, here we restricted to $k=1$ in order to compare to the $k=1$ ABJM theory, which is also dual to $\mathrm{AdS}_{4} \times S^{7}$ (the point being that the $\mathbb{Z}_{k}$ quotients in each case are different). In fact the general $k$ case is $a=k, b=0$ of section 4.3 .
} 


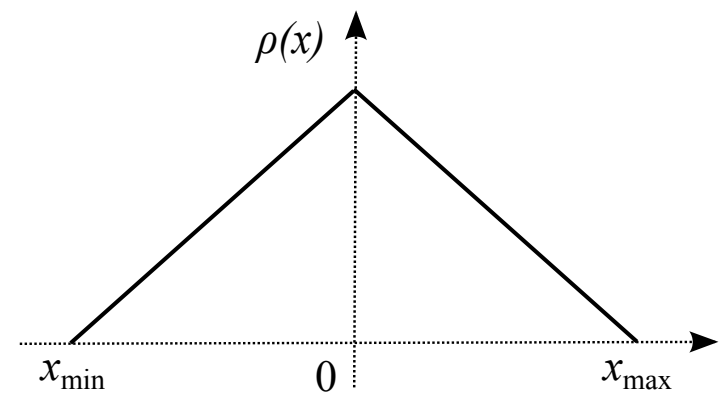

Figure 3. Eigenvalue density as a function of $x$. There are three points where $\rho^{\prime}(x)$ is discontinuous, corresponding to critical points of $h_{M}$.

discontinuous at the endpoints and at the point $x=0$, which arises as the image of the D6-brane locus under $h_{M}$. The Wilson loop is again given by (4.8), with $k=1$.

\subsection{Dual to $Q^{1,1,1} / \mathbb{Z}_{k}$}

Our next example is that of the homogeneous (and toric) Sasaki-Einstein manifold $Q^{1,1,1} / \mathbb{Z}_{k}$. The manifold $Q^{1,1,1}$ is the total space of an $S^{1}$ fibration over the product of three copies of $S^{2}$, i.e. $S^{1} \hookrightarrow Q^{1,1,1} \rightarrow S^{2} \times S^{2} \times S^{2}$, which describes its structure as a regular Sasaki-Einstein manifold. Even though this manifold is toric, and the geometrical techniques described in section 3.5 can be applied, we will instead take advantage of the fact that the metric is known explicitly on this space.

The Sasaki-Einstein metric on $Q^{1,1,1}$ can be written as

$$
g_{Y_{7}}=\frac{1}{16}\left(\mathrm{~d} \psi+\sum_{i=1}^{3} \cos \theta_{i} \mathrm{~d} \varphi_{i}\right)^{2}+\frac{1}{8} \sum_{i=1}^{3}\left(\mathrm{~d} \theta_{i}^{2}+\sin ^{2} \theta_{i} \mathrm{~d} \varphi_{i}^{2}\right),
$$

where the coordinates $\theta_{i} \in[0, \pi]$ and $\varphi_{i} \in[0,2 \pi)$ are the usual $S^{2}$ coordinates, and the coordinate $\psi \in[0,4 \pi)$ parametrizes the $S^{1}$ fibre. The contact form is simply

$$
\eta=\frac{1}{4}\left(\mathrm{~d} \psi+\sum_{i=1}^{3} \cos \theta_{i} \mathrm{~d} \varphi_{i}\right)
$$

and for the field theory model below the M-theory circle is generated by $\zeta_{M}=\frac{1}{k}\left(\partial_{\varphi_{1}}+\partial_{\varphi_{2}}\right)$. The M-theory Hamiltonian follows straightforwardly and reads

$$
h_{M}=\eta\left(\zeta_{M}\right)=\frac{1}{4 k}\left(\cos \theta_{1}+\cos \theta_{2}\right) .
$$

The length of a supersymmetric M-theory circle is always given by $2 \pi h_{M}(\hat{p})$, where $\hat{p} \in$ $Y_{7}$ covers a fixed point $p$ of $\xi$, with $p \in M_{6}=Y_{7} / \mathrm{U}(1)_{M}$. However, when the Sasaki-Einstein manifold is regular, as in the case at hand, we may also describe the supersymmetric Mtheory circles in terms of the base Kähler-Einstein manifold $B_{6}=Y_{7} / \mathrm{U}(1)_{R}$, where $\mathrm{U}(1)_{R}$ is generated by the Reeb vector $\xi$. In this point of view, the supersymmetric M-theory circles cover fixed points of $\zeta_{M}$ on $B_{6}$, which in the case at hand is $B_{6}=S^{2} \times S^{2} \times S^{2}$ because $\xi=4 \partial_{\psi}$. These points are located at $\left\{\left(\theta_{1}, \theta_{2}\right) \mid \theta_{1} \in\{0, \pi\}, \theta_{2} \in\{0, \pi\}\right\}$. Thus 


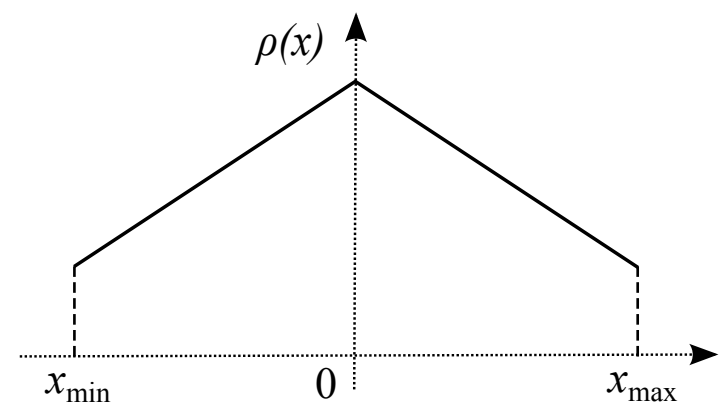

Figure 4. Eigenvalue density $\rho(x)$. There are three points where $\rho^{\prime}(x)$ is discontinuous, associated with supersymmetric M-theory circles.

one obtains three critical values $c_{1}=c_{\min }=-\frac{1}{2 k}, c_{2}=0, c_{3}=c_{\max }=\frac{1}{2 k}$. Notice these are $S^{2}$ loci of critical points, parametrized by $\left(\theta_{3}, \varphi_{3}\right)$.

Being Einstein, the contact volume of $Q^{1,1,1} / \mathbb{Z}_{k}$ is equal to the Riemannian volume, with

$$
\operatorname{Vol}\left(Q^{1,1,1} / \mathbb{Z}_{k}\right)=\frac{\pi^{4}}{8 k}
$$

and as usual the $\mathbb{Z}_{k}$ quotient is along $\mathrm{U}(1)_{M}$ generated by $\zeta_{M}$. The general formula (1.6) tells us that the matrix model variable $x_{\max }=-x_{\min }$ predicted from the gravity calculation is

$$
x_{\max }=\frac{(2 \pi)^{3}}{\sqrt{96 \operatorname{Vol}\left(Q^{1,1,1} / \mathbb{Z}_{k}\right)}} c_{\max }=\frac{2 \pi}{\sqrt{3 k}} .
$$

A dual field theory to $Q^{1,1,1} / \mathbb{Z}_{k}$ has been proposed in $[11,13]$. This theory is closely related to the ABJM theory. In addition to the bifundamental fields $A_{i}, B_{i}$, a pair of field in the (anti-) fundamental representation is added to each gauge group node, and one adds a cubic term to the superpotential

$$
\mathcal{W}_{\text {cubic }}=\operatorname{Tr}\left(q_{1} A_{1} \tilde{q}_{1}+q_{2} A_{2} \tilde{q}_{2}\right)
$$

The corresponding matrix model has been worked out in [32], where it was found that the density of the real part of the eigenvalues is

$$
\rho(x)=\frac{k}{4 \pi^{2}}\left(2 x_{\max }-|x|\right) \quad \text { for } \quad x_{\min }<x<x_{\max },
$$

with $x_{\max }=\frac{2 \pi}{\sqrt{3 k}}$, thus agreeing with (4.19). Moreover, the derivative of $\rho$ is discontinuous at the endpoints and at the point $x=0$, as predicted by $c_{1}, c_{2}$ and $c_{3}$ above. The Wilson loop calculated from the field theory then agrees with the gravity computation, and reads

$$
\log \langle W\rangle=\frac{2 \pi}{\sqrt{3 k}} N^{1 / 2} .
$$

\section{3 $\mathcal{N}=8$ super-Yang-Mills with flavour}

In this section we consider a family of $\mathcal{N}=2$ Chern-Simons-matter theories that generalize the mirror to the ABJM theory discussed in section 4.1.2. These were discussed in section 4 of [33], having been first introduced in [13]. 
One begins with $\mathcal{N}=8$ super-Yang-Mills with gauge group $\mathrm{U}(N)$, which is the theory on $N$ D2-branes in flat space. In $\mathcal{N}=2$ language we have three adjoint chiral matter fields $X_{1}, X_{2}, X_{3}$, together with the cubic superpotential $\operatorname{Tr} X_{3}\left[X_{1}, X_{2}\right]$. To this we add matter fields in the fundamental and anti-fundamental representations, which breaks the supersymmetry generically to $\mathcal{N}=2$. More precisely, we add $n_{1}$ fields $\left(q_{j}^{(1)}, \tilde{q}_{j}^{(1)}\right), n_{2}$ fields $\left(q_{j}^{(2)}, \tilde{q}_{j}^{(2)}\right)$ and $n_{3}$ fields $\left(q_{j}^{(3)}, \tilde{q}_{j}^{(3)}\right)$, together with the cubic superpotential

$$
\mathcal{W}=\operatorname{Tr}\left[\sum_{j=1}^{n_{1}} q_{j}^{(1)} X_{1} \tilde{q}_{j}^{(1)}+\sum_{j=1}^{n_{2}} q_{j}^{(2)} X_{2} \tilde{q}_{j}^{(2)}+\sum_{j=1}^{n_{3}} q_{j}^{(3)} X_{3} \tilde{q}_{j}^{(3)}+X_{3}\left[X_{1}, X_{2}\right]\right],
$$

so that the theory in section 4.1.2 is simply $n_{1}=1, n_{2}=n_{3}=0$. As for the mirror to the ABJM theory, the additional $(q, \tilde{q})$ fields will arise in type IIA from strings stretching from the D2-branes to D6-brane loci.

In [13] it was shown that the (quantum corrected) moduli space of vacua of these theories, for $N=1$, may be parametrized by the three coordinates $X_{1}, X_{2}, X_{3}$, together with the monopole operators $T, \tilde{T}$, which satisfy the constraint

$$
T \tilde{T}=X_{1}^{n_{1}} X_{2}^{n_{2}} X_{3}^{n_{3}}
$$

This defines a Calabi-Yau cone $C\left(Y_{7}\right)$ as a hypersurface singularity in $\mathbb{C}^{5}$. The M-theory circle is straightforward to identify in this case, since by definition the monopole operators $T, \tilde{T}$ have charges \pm 1 , respectively, under $\mathrm{U}(1)_{M}$, while the $X_{i}$ are uncharged. Notice this implies that the quotient $C\left(Y_{7}\right) / \mathbb{C}_{M}^{*} \cong \mathbb{C}^{3}$ by the complexified M-theory circle (defined as a GIT quotient) is simply $\mathbb{C}^{3}$, parametrized by $X_{1}, X_{2}, X_{3}$, which implies that the type IIA description involves $N$ D2-branes in flat space. Moreover, $\mathrm{U}(1)_{M}$ fixes $T=\tilde{T}=0$, which defines the surface $\left\{X_{1}^{n_{1}} X_{2}^{n_{2}} X_{3}^{n_{3}}=0\right\} \subset \mathbb{C}^{3}$, which becomes a D6-brane locus. This then geometrically engineers the gauge theory described above, with superpotential (4.23).

It is straightforward to analyse the matrix model for this gauge theory, as described in section 2.2 and carried out in [33]. The eigenvalue density is given by

$$
\rho(x)= \begin{cases}\frac{\left(\sum_{i=1}^{3} n_{i} \Delta_{i}-2 \Delta_{m}\right)}{8 \pi^{2} \Delta_{1} \Delta_{2} \Delta_{3}}\left(x-x_{\min }\right), & x_{\min }<x<0 \\ \frac{\left(\sum_{i=1}^{3} n_{i} \Delta_{i}+2 \Delta_{m}\right)}{8 \pi^{2} \Delta_{1} \Delta_{2} \Delta_{3}}\left(x_{\max }-x\right), & 0<x<x_{\max }\end{cases}
$$

and the endpoints are

$$
x_{\max / \min }= \pm \sqrt{\frac{8 \pi^{2} \Delta_{1} \Delta_{2} \Delta_{3}\left(\sum_{i=1}^{3} n_{i} \Delta_{i} \mp 2 \Delta_{m}\right)}{\left(\sum_{i=1}^{3} n_{i} \Delta_{i}\right)\left(\sum_{i=1}^{3} n_{i} \Delta_{i} \pm 2 \Delta_{m}\right)}} .
$$

Here $\Delta_{i}=\Delta\left(X_{i}\right), i=1,2,3$, are the R-charges of the fields $X_{i}$, while $\Delta_{m}=\Delta(T)=\Delta(\tilde{T})$ is the R-charge of the monopole operators. As described in section 2.2, these may be left a priori arbitrary at this point, the only restriction being that the superpotential $\mathcal{W}$ has R-charge $\Delta(\mathcal{W})=2$. This leads to the constraint $\sum_{i=1}^{3} \Delta_{i}=2$. The shape of $\rho$ as a function of $x$ is shown in figure 5 . 


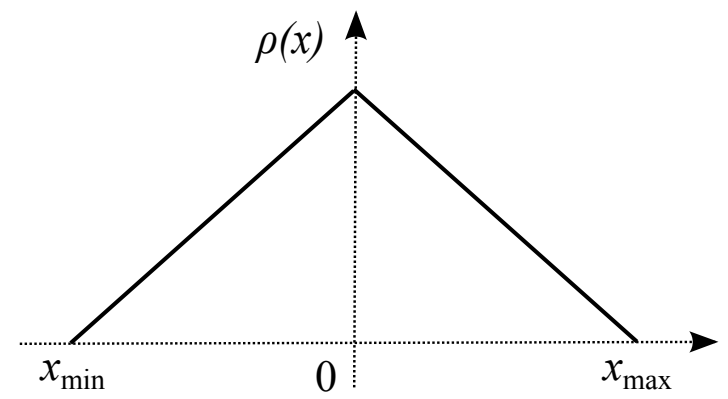

Figure 5. Eigenvalue density as a function of $x$. There are three points where $\rho^{\prime}(x)$ is discontinuous, and we correspondingly expect to find three critical points of $h_{M}$, with associated supersymmetric circles.

The superconformal R-charges are determined by maximizing the free energy $F$ as a function of the R-charges. This immediately leads to $\Delta_{m}=0$, and then

$$
F=\frac{2 \sqrt{2} \pi \sqrt{\Delta_{1} \Delta_{2} \Delta_{3}\left(\sum_{i=1}^{3} n_{i} \Delta_{i}\right)}}{3} N^{3 / 2},
$$

which must be further maximized subject to the constraint $\sum_{i=1}^{3} \Delta_{i}=2$. In practice the formulae are rather too unwieldy for general $n_{i}$, so following [33] we restrict to the case $n_{1}=a, n_{2}=b, n_{3}=0$. In this case the free energy is maximized by

$$
\Delta_{1}=\frac{a-2 b+\sqrt{a^{2}+b^{2}-a b}}{2(a-b)}, \quad \Delta_{2}=\frac{b-2 a+\sqrt{a^{2}+b^{2}-a b}}{2(b-a)}, \quad \Delta_{3}=\frac{1}{2},
$$

and thus

$$
x_{\max / \min }= \pm 2 \pi \sqrt{\frac{\Delta_{1} \Delta_{2}}{a \Delta_{1}+b \Delta_{2}}} .
$$

The moduli space equation (4.24) correspondingly reduces to $T \tilde{T}=X_{1}^{a} X_{2}^{b}$. The field $X_{3}$ is then unconstrained, and the Calabi-Yau cone takes the product form $C\left(Y_{7}\right)=$ $\mathbb{C} \times C\left(Y_{5}\right)$, where $X_{3}$ is a coordinate on $\mathbb{C}$ and $C\left(Y_{5}\right)$ is precisely the $Y_{5}=L^{a, b, a}$ toric singularity. ${ }^{10}$ The toric diagram has lattice vectors

$$
\begin{array}{ll}
w_{1}=(0,0,0), & w_{2}=(0,1,0), \\
w_{4}=(0,0, a), & w_{5}=(0,1, b),
\end{array}
$$

and is shown in figure 6 . Recall that we parametrize the Reeb vector by $\xi=\left(4, \xi_{2}, \xi_{3}, \xi_{4}\right)$, and that the four-dimensional outward-pointing vectors to the facets are $v_{a}=\left(1, w_{a}\right)$. With the method described earlier in section 3.5, the volume of the base $Y_{7}$ and the Reeb vector can be found and expressed in terms of $\Delta_{1}$ and $\Delta_{2}$, and one finds

$$
\operatorname{Vol}\left(Y_{7}\right)=\frac{\pi^{4}}{6} \frac{1}{\Delta_{1} \Delta_{2}\left(a \Delta_{1}+b \Delta_{2}\right)},
$$

\footnotetext{
${ }^{10} \mathrm{~A}$ discussion of the relation between the hypersurface and toric geometry descriptions of this three-fold singularity may be found in [45].
} 


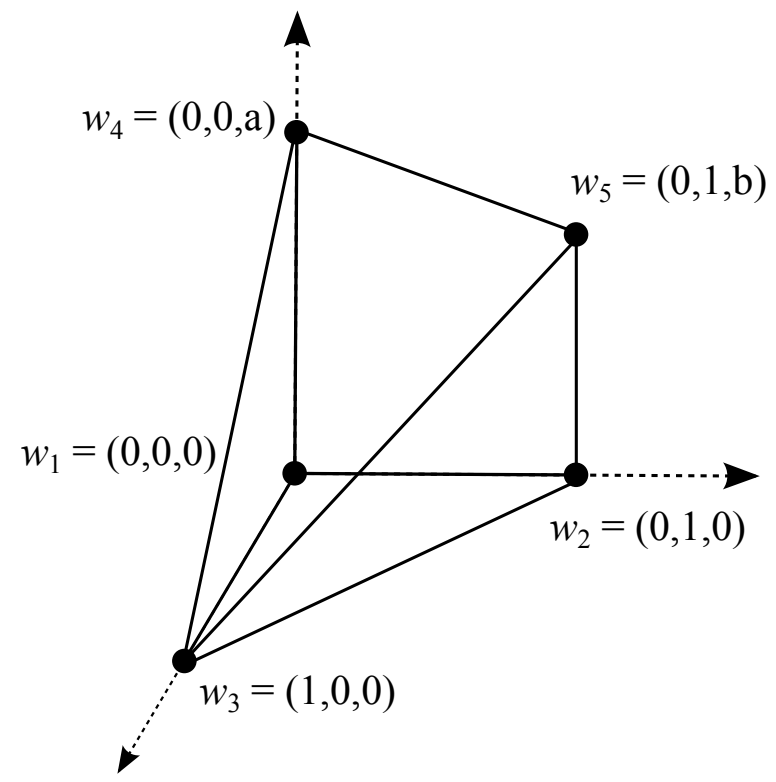

Figure 6. Toric diagram corresponding to $C\left(Y_{7}\right)=\left\{T \tilde{T}=X_{1}^{a} X_{2}^{b}\right\} \times \mathbb{C}$. The apex is not an isolated singularity, as one sees from the non-triangular face with vertices $(1,2,4,5)$.

and

$$
\vec{\xi}=\left(4,1,2 \Delta_{2}, a \Delta_{1}+b \Delta_{2}\right)
$$

The M-theory circle in this basis is given by $\zeta_{M}=(0,0,0,-1)$; one can derive this by writing the functions $T, \tilde{T}, X_{i}$ in terms of the toric geometry formalism above (see, for example, section 4.3 of [45]). Recall also that in this formalism the M-theory Hamiltonian function is given by (3.33). Thus in this case we have simply $h_{M}=-2 y_{4}$. The critical points of $h_{M}$ must always lie on the boundary of the polyhedral cone, which are coordinate singularities, and thus it is easiest to determine this critical set using the method described at the end of section 3.5. We denote the face of the toric diagram which has vertices $\left\{v_{a}, v_{b}, v_{c}, \ldots\right\}$ by $(a, b, c, \ldots)$. Equation (3.34) then has two types of solution:

$$
\begin{array}{rlrl}
h_{M} & =0 & \text { on } \quad(2,3,5),(1,3,4),(1,2,4,5), \\
\left|h_{M}\right| & =\frac{1}{a \Delta_{1}+b \Delta_{2}} \quad \text { on } \quad(1,2,3),(3,4,5),
\end{array}
$$

and correspondingly one has the critical values $h_{M}=c_{i}$ given by

$$
c_{3}=-c_{1}=\frac{1}{a \Delta_{1}+b \Delta_{2}}, \text { and } \quad c_{2}=0 \text {. }
$$

Notice here that the face $(1,2,4,5)$ (being non-triangular) corresponds to the $S^{1}$ locus of $L^{a, b, a}$ conical singularities in $Y_{7}$. Using the general formula (1.6) we then find that these values of $c_{i}$ precisely match the corresponding positions $x_{1}, x_{2}, x_{3}$ at which the derivative of the eigenvalue density $\rho^{\prime}(x)$ is discontinuous. Finally, using (1.4) the Wilson loop is

$$
\log \langle W\rangle_{\text {gravity }}=2 \pi \sqrt{\frac{\Delta_{1} \Delta_{2}}{a \Delta_{1}+b \Delta_{2}}} N^{1 / 2}=x_{\max } N^{1 / 2}=\log \langle W\rangle_{\mathrm{QFT}},
$$

where we used (4.29). 


\section{4 $L^{a, 2 a, a}$ Chern-Simons-quivers}

In this section and the next we study two families of examples whose matrix models were first analyzed in [37].

The $\mathcal{N}=2$ field theories begin life as low-energy theories on $N$ D2-branes at an $L^{a, b, a}$ Calabi-Yau three-fold singularity. This may be simply described as the hypersurface $\left\{w z=u^{a} v^{b}\right\} \subset \mathbb{C}^{4}$, where $(w, z, u, v)$ are the coordinates on $\mathbb{C}^{4}$. This geometry also appeared in the previous subsection of course, but there the M-theory Calabi-Yau four-fold was a product $\mathbb{C} \times C\left(L^{a, b, a}\right)$, whereas here instead $C\left(L^{a, b, a}\right)$ arises as the type IIA spacetime. The low-energy theory on the $N$ D2-branes is known from [57-59], and is described by a $\mathrm{U}(N)^{a+b}$ quiver gauge theory, with a superpotential $\mathcal{W}$ consisting of both cubic and quartic terms in the bifundamental and adjoint chiral matter fields. Without loss of generality we may take $b \geq a$, in which case there are $b-a$ adjoint chiral superfields associated to $b-a$ of the $a+b \mathrm{U}(N)$ gauge group factors, and a total of $2(a+b)$ bifundamental fields. We refer the reader to the above references for further details of these quiver gauge theories.

The D2-brane theories become M2-brane theories at a Calabi-Yau four-fold by turning on RR flux in the type IIA background, following [12] and in particular the construction in [6]. Geometrically this fibres the M-theory circle over the base $C\left(L^{a, b, a}\right)$, and in the field theory introduces Chern-Simons couplings for the gauge group, described by a vector of Chern-Simons levels $\vec{k}=\left(k_{1}, \ldots, k_{a+b}\right)=\left(k_{1}, \ldots, k_{b-a} \| k_{b-a+1}, \ldots, k_{a+b}\right)$, where the double bar separates the copies of $\mathrm{U}(N)$ with adjoint fields from those without. This construction is described in more detail in [37].

Our first class of examples arise from $L^{a, 2 a, a}$ quiver theories, where the vector of ChernSimons levels is $\vec{k}=(0, \ldots, 0,-2 k \| k, k,-k, k,-k, \ldots, k,-k, k)$, with $k \in \mathbb{Z}$. These theories generalize the model first studied in [31]. The matrix model may be solved using the general large $N$ saddle point method described in section 2.2, and one finds [37] the eigenvalue density

$$
\rho(x)=\left\{\begin{array}{lc}
\frac{4 a k \pi x(1-\Delta)+\mu}{16 a \pi^{3}(1-\Delta) \Delta^{2}}, & -\frac{\mu}{4 a k \pi(1-\Delta)}<x<-\frac{\mu}{2 a k \pi(2-\Delta)} \\
\frac{\mu}{16 a \pi^{3} \Delta(2-\Delta)(1-\Delta)}, & -\frac{\mu}{2 a k \pi(2-\Delta)}<x<\frac{\mu}{2 a k \pi(2-\Delta)} \\
-\frac{4 a k \pi x(1-\Delta)-\mu}{16 a \pi^{3}(1-\Delta) \Delta^{2}}, & \frac{\mu}{2 a k \pi(2-\Delta)}<x<\frac{\mu}{4 a k \pi(1-\Delta)}
\end{array},\right.
$$

where we have defined ${ }^{11}$

$$
\mu=8 a \pi^{2} \sqrt{\frac{k \Delta\left(2-3 \Delta+\Delta^{2}\right)^{2}}{4-3 \Delta}} .
$$

Here the single R-charge variable $\Delta$ parametrizes the R-charges of all the chiral matter fields, as in [37]. The eigenvalue density $\rho(x)$ is shown in figure 7.

The free energy, as a function of $\Delta$, is given by

$$
F=\frac{8 a \pi}{3} \sqrt{\frac{k \Delta(1-\Delta)^{2}(2-\Delta)^{2}}{(4-3 \Delta)}} N^{3 / 2} .
$$

\footnotetext{
${ }^{11}$ The variable $\mu$ arises as a Lagrange multiplier, enforcing that $\rho(x)$ is a density satisfying (2.13).
} 


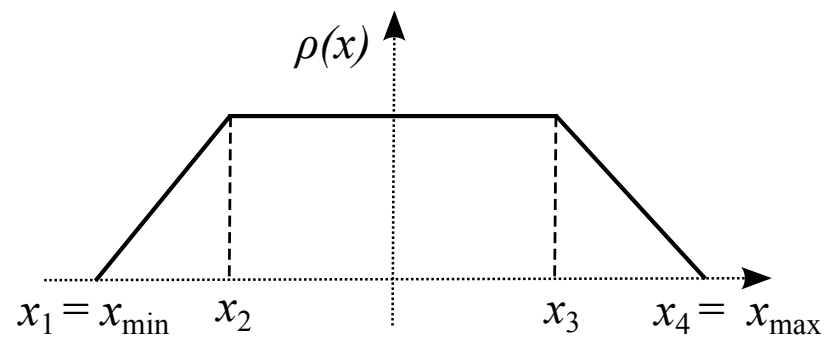

Figure 7. Eigenvalue density as a function of $x$. There are 4 points $x_{1}, x_{2}, x_{3}, x_{4}$ where $\rho^{\prime}(x)$ is discontinuous, corresponding to critical points of $h_{M}$.

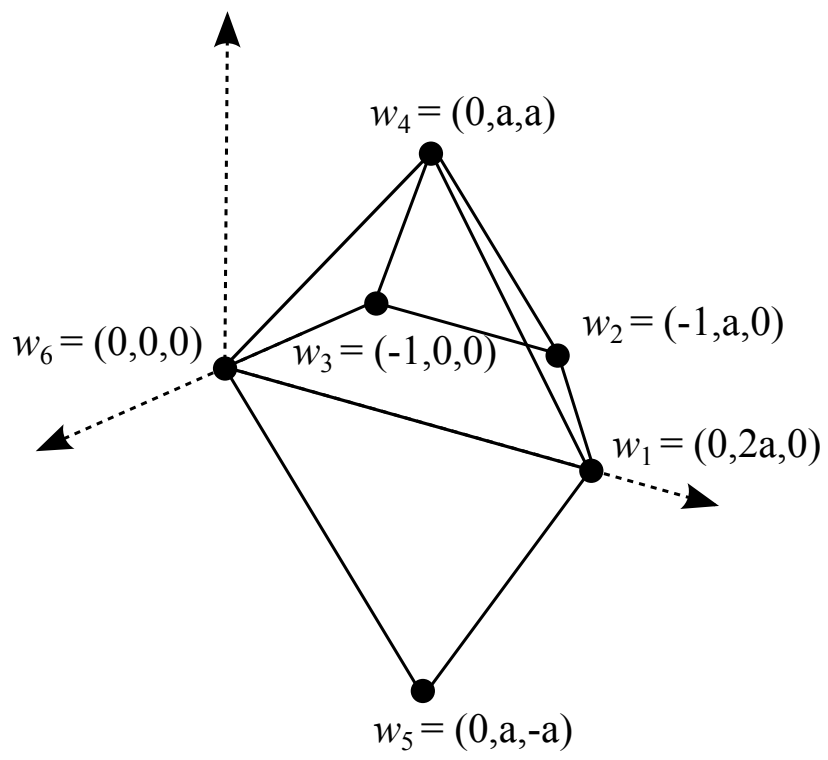

Figure 8. Toric diagram of the $L^{a, 2 a, a}$ Chern-Simons-quiver theories with $k=1$.

One may then maximize $F$ to determine the superconformal $\Delta$, finding the cubic irrational

$$
\Delta=\frac{1}{18}\left[19-\frac{37}{(431-18 \sqrt{417})^{1 / 3}}-(431-18 \sqrt{417})^{1 / 3}\right] \simeq 0.319 .
$$

This agrees with the value computed in [31], which was for the particular case $a=1$.

Turning to the dual geometry, the Calabi-Yau four-fold that arises as the Abelian $N=1$ moduli space of these theories has toric data (for $k=1$ )

$$
\begin{array}{lll}
w_{1}=(0,2 a, 0), & w_{2}=(-1, a, 0), & w_{3}=(-1,0,0), \\
w_{4}=(0, a, a), & w_{5}=(0, a,-a), & w_{6}=(0,0,0),
\end{array}
$$

and with toric diagram shown in figure 8. The volume of $Y_{7}$ may be computed as described in section 3.5 , and one obtains

$$
\operatorname{Vol}\left(Y_{7}\right)=\frac{\pi^{4}(4-3 \Delta)}{96 a^{2} k \Delta(\Delta-1)^{2}(\Delta-2)^{2}}
$$


with corresponding Reeb vector field

$$
\vec{\xi}=(4,-4 \Delta, 2 a(2-\Delta), 0) .
$$

The M-theory circle for this field theory is given in this basis by $\zeta_{M}=(0,0,0,1)$, so that $Y_{7}$ is given by a $\mathbb{Z}_{k}$ quotient of the geometry appearing in figure 8 , with $\mathbb{Z}_{k} \subset \mathrm{U}(1)_{M}$. We may again compute the critical points of the M-theory Hamiltonian $h_{M}=2 y_{4}$ using the method at the end of section 3.5. Equation (3.34) has solutions associated to the following faces of the toric diagram:

$$
\begin{aligned}
\left(h_{M}=0\right. & \text { on } \quad(1,4,5,6)), \\
\left|h_{M}\right| & =\frac{1}{4 a(1-\Delta)} \quad \text { on } \quad(2,3,4),(2,3,5), \\
\left|h_{M}\right| & =\frac{1}{2 a(2-\Delta)} \quad \text { on } \quad(1,2,4),(1,2,5),(3,4,6),(3,5,6),
\end{aligned}
$$

and correspondingly one has the critical values $h_{M}=c_{i}$ given by

$$
c_{4}=-c_{1}=\frac{1}{4 a k(1-\Delta)}, \quad \text { and } \quad c_{3}=-c_{2}=\frac{1}{2 k a(2-\Delta)} .
$$

Note here that the face $(1,4,5,6)$ describes a singular $S^{1}$ locus in $Y_{7}$, and thus although $h_{M}=0$ here, formally leading to zero-action M2-branes, the tangent space is singular. Using the general formula (1.6) we then find that these values of $c_{i}$ precisely match the corresponding positions $x_{1}, x_{2}, x_{3}, x_{4}$ at which the derivative of the eigenvalue density $\rho^{\prime}(x)$ is discontinuous. Explicitly, the actions of M2-branes wrapped on the corresponding calibrated $S^{1} \subset Y_{7}$ are then

$$
\begin{array}{r}
-S_{\mathrm{M} 2}\left(c_{2}\right)=4 \pi(1-\Delta) \sqrt{\frac{\Delta}{k(4-3 \Delta)}} N^{1 / 2}, \\
\log \langle W\rangle=-S_{\mathrm{M} 2}\left(c_{4}\right)=2 \pi(2-\Delta) \sqrt{\frac{\Delta}{k(4-3 \Delta)}} N^{1 / 2},
\end{array}
$$

with the latter determining the Wilson loop VEV, and showing that the field theory and gravity computations of it agree.

\section{5 $L^{a, b, a}$ Chern-Simons-quivers}

Our second family within this class are the $L^{a, b, a}$ Chern-Simons-quiver theories, with the vector of Chern-Simons levels now given by $\vec{k}=(0, \ldots, k,-2 k \| k, 0, \ldots, 0)$. One finds the eigenvalue density [37]

$$
\rho(x)=\left\{\begin{array}{lc}
\frac{4 k \pi x(1-\Delta)+\mu}{16 \pi^{3}(1-\Delta) \Delta((b-2)(1-\Delta)+a \Delta)}, & -\frac{\mu}{4 k \pi(1-\Delta)}<x<-\frac{\mu}{2 k \pi(b(1-\Delta)+a \Delta)} \\
\frac{\mu}{16 \pi^{3}(1-\Delta) \Delta(b(1-\Delta)+a \Delta)}, & -\frac{\mu}{2 k \pi(b(1-\Delta)+a \Delta)}<x<\frac{\mu}{2 k \pi(b(1-\Delta)+a \Delta)} \\
-\frac{4 k \pi x(1-\Delta)-\mu}{16 \pi^{3}(1-\Delta) \Delta((b-2)(1-\Delta)+a \Delta)}, & \frac{\mu}{2 k \pi(b(1-\Delta)+a \Delta)}<x<\frac{\mu}{4 k \pi(1-\Delta)}
\end{array}\right.
$$




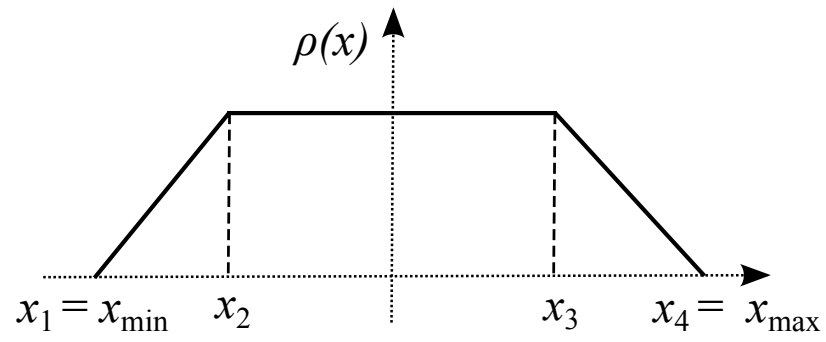

Figure 9. Eigenvalue density as a function of $x$. There are again 4 points $x_{1}, x_{2}, x_{3}, x_{4}$ where $\rho^{\prime}(x)$ is discontinuous, corresponding to critical points of $h_{M}$.

where we have defined

$$
\mu=8 \pi^{2} \sqrt{\frac{k \Delta(1-\Delta)^{2}(b(1-\Delta)+a \Delta)^{2}}{(b-2)(1-\Delta)+a \Delta}} .
$$

Again, the R-charge variable $\Delta$ parametrizes the $\mathrm{R}$-charges of all the chiral matter fields, as detailed in [37]. The eigenvalue density $\rho(x)$ is shown in figure 9 .

The free energy, as a function of $\Delta$, is given by

$$
F=\frac{8 \pi}{3} \sqrt{\frac{k(1-\Delta)^{2} \Delta(b(1-\Delta)+a \Delta)^{2}}{(b+2)(1-\Delta)+a \Delta}} N^{3 / 2} .
$$

One may then maximize $F$ to find an expression (not presented) for the superconformal $\Delta$ that depends on $a$ and $b$.

The corresponding Calabi-Yau four-fold that arises as the Abelian $N=1$ moduli space of these theories has toric data (for $k=1$ )

$$
\begin{array}{llll}
w_{1}=(0,0,0), & w_{2}=(1,-1,0), & w_{3}=(1,1,0), & w_{4}=(b-1,-1,0), \\
w_{5}=(b-1,1,0), & w_{6}=(b, 0,0), & w_{7}=(0,0,1), & w_{8}=(a, 0,1),
\end{array}
$$

and with toric diagram shown in figure 10. The volume of $Y_{7}$ may be computed as described in section 3.5 , and one obtains

$$
\operatorname{Vol}\left(Y_{7}\right)=\frac{\pi^{4}((b+2)(1-\Delta)+a \Delta)}{96 k \Delta(1-\Delta)^{2}(b(1-\Delta)+a \Delta)^{2}}
$$

with corresponding Reeb vector

$$
\vec{\xi}=(4,2(b(1-\Delta)+a \Delta), 0,4 \Delta) .
$$

The M-theory circle for this field theory is given in this basis by $\zeta_{M}=(0,0,1,0)$, so that again $Y_{7}$ is given by a $\mathbb{Z}_{k}$ quotient of the geometry appearing in figure 10, with $\mathbb{Z}_{k} \subset \mathrm{U}(1)_{M}$. The M-theory Hamiltonian is $h_{M}=2 y_{3}$, and its critical points may be computed from equation (3.34), which has solutions on the following faces of the toric 


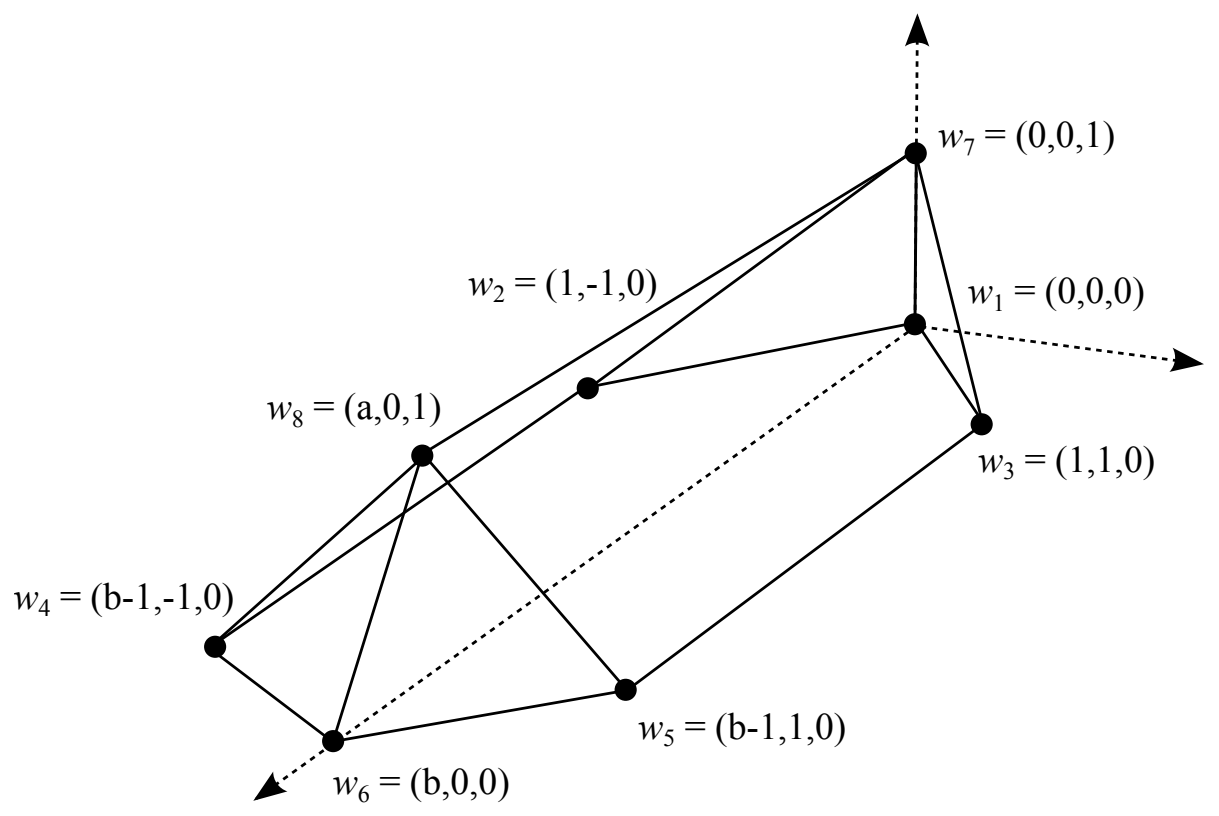

Figure 10. Toric diagram of the $L^{a, b, a}$ Chern-Simons-quiver theories with $k=1$.

diagram:

$$
\begin{array}{lll}
\left(h_{M}=0\right. & \text { on } \quad(1,2,3,4,5,6),) \\
\left|h_{M}\right|=\frac{1}{4(1-\Delta)} & \text { on } \quad(2,4,7,8),(3,5,7,8), \\
\left|h_{M}\right|=\frac{1}{2(b(1-\Delta)+a \Delta)} & \text { on } \quad(1,2,7),(1,3,7),(4,6,8),(5,6,8),
\end{array}
$$

and correspondingly one has critical values $h_{M}=c_{i}$ given by

$$
c_{4}=-c_{1}=\frac{1}{4 k(1-\Delta)}, \quad \text { and } \quad c_{3}=-c_{2}=\frac{1}{2 k(b(1-\Delta)+a \Delta)} .
$$

Using the general formula (1.6) we then find that these values of $c_{i}$ precisely match the corresponding positions $x_{1}, x_{2}, x_{3}, x_{4}$ at which the derivative of the eigenvalue density $\rho^{\prime}(x)$ is discontinuous. Explicitly, the actions of M2-branes wrapped on the corresponding calibrated $S^{1} \subset Y_{7}$ are

$$
\begin{gathered}
-S_{\mathrm{M} 2}\left(c_{2}\right)=4 \pi(1-\Delta) \sqrt{\frac{\Delta}{k((2+b)(1-\Delta)+a \Delta)} N^{1 / 2},} \\
\log \langle W\rangle=-S_{\mathrm{M} 2}\left(c_{4}\right)=2 \pi(b(1-\Delta+a \Delta)) \sqrt{\frac{\Delta}{k((2+b)(1-\Delta)+a \Delta)}} N^{1 / 2},
\end{gathered}
$$

with the latter determining the Wilson loop VEV, and showing that the field theory and gravity computations of it agree. 


\subsection{Duals to non-Einstein solutions with flux}

In this final class of examples we examine a family of $\mathcal{N}=2$ superconformal theories which are dual to warped non-Einstein solutions, with non-zero internal $F_{4}$ flux in (3.1). As we described in section 3.1, solutions with non-zero M2-brane charge $N$ still have a contact structure, and our formalism then applies.

Following [33], we begin with the $\mathcal{N}=8$ super-Yang-Mills theories with flavour in section 4.3, for which the dual Calabi-Yau four-fold is $C\left(Y_{7}\right)=\mathbb{C} \times C\left(L^{a, b, a}\right)$. The field theories have a free chiral field $X_{3}$, of scaling dimension $\Delta\left(X_{3}\right)=\Delta_{3}=\frac{1}{2}$, and we consider perturbing the theory by adding the deformation $\lambda X_{3}^{p}$ to the superpotential. In three dimensions this is a relevant deformation for $p=2$ and $p=3$. The gravity dual to the resulting infrared (IR) fixed point of the massive $p=2$ deformation is the Corrado-PilchWaner solution of [20], while the supergravity dual to the cubic $p=3$ deformation was found only recently (independently) in [25,60]. In both cases these are warped $\mathrm{AdS}_{4} \times Y_{7}$ solutions with flux, where crucially $Y_{7}$ has the same topology as before the deformation, and the toric symplectic structure of the cone $C\left(Y_{7}\right)$ is also the same. However, the Reeb vector field (and hence also contact structure) is different.

This deformation was studied in the matrix model in [33], where they found the universal behaviour

$$
\frac{F^{\mathrm{IR}}}{F^{\mathrm{UV}}}=\frac{16(p-1)^{3 / 2}}{3 \sqrt{3} p^{2}}
$$

relating the free energies $F$ of the IR and UV theories. One correspondingly finds that the scaling dimensions $\Delta_{i}=\Delta\left(X_{i}\right)$ are related as

$$
\frac{\Delta_{1}^{\mathrm{IR}}}{\Delta_{1}^{\mathrm{UV}}}=\frac{4(p-1)}{3 p}=\frac{\Delta_{2}^{\mathrm{IR}}}{\Delta_{2}^{\mathrm{UV}}}, \quad \Delta_{3}^{\mathrm{IR}}=\frac{2}{p},
$$

and that the eigenvalue distribution takes the same form as before, with $x_{\max }=-x_{\min }$, but now with the endpoints rescaled as

$$
\frac{x_{\max }^{\mathrm{IR}}}{x_{\max }^{\mathrm{UV}}}=\frac{4 \sqrt{p-1}}{\sqrt{3} p} .
$$

As explained in general in [25], using (2.16) the field theory result (4.56) matches with the supergravity result relating the (contact) volumes of the IR and UV solutions:

$$
\frac{\operatorname{Vol}_{\eta}\left(Y_{7}^{\mathrm{IR}}\right)}{\operatorname{Vol}\left(Y_{7}^{\mathrm{UV}}\right)}=\frac{27 p^{4}}{256(p-1)^{3}} \equiv V(p)
$$

This result was derived in [25] using the contact geometry of the IR solutions. It is crucial that here the volume is the contact volume $\mathrm{Vol}_{\eta}$ and not the Riemannian volume (which is different). The Reeb vector field of the UV Calabi-Yau geometry $\mathbb{C} \times C\left(Y_{5}\right)$ may be written as

$$
\xi^{\mathrm{UV}}=\partial_{\phi_{3}}+\partial_{\varphi}
$$


where $\partial_{\phi_{3}}$ rotates the free field $X_{3}$ with charge 1 (so $X_{3}=\left|X_{3}\right| \mathrm{e}^{\mathrm{i} \phi_{3}}$ ), while $\partial_{\varphi}$ denotes the Reeb vector field of the Calabi-Yau three-fold cone $C\left(Y_{5}\right)$. As shown in [25], the IR Reeb vector field for the solution with flux is then

$$
\xi^{\mathrm{IR}}=\frac{4}{p} \partial_{\phi_{3}}+\frac{4(p-1)}{3 p} \partial_{\varphi}
$$

In fact these formulae are directly related to the rescalings of the R-charges in (4.57). Using this simple rescaling one can check that the M2-brane actions are rescaled as

$$
\frac{S_{\mathrm{M} 2}^{\mathrm{IR}}}{S_{\mathrm{M} 2}^{\mathrm{UV}}}=\frac{\frac{3 p}{4(p-1)}}{\sqrt{V(p)}}=\frac{4 \sqrt{p-1}}{\sqrt{3} p},
$$

thus matching the field theory result (4.58). It of course follows immediately that the field theory and gravity results for the Wilson loop VEV agree. What is remarkable here is that we are able to compute this so straightforwardly, even though the $p=3$ supergravity solution is known only numerically in $[25,60]$. Of course, it is precisely because we know the contact structure explicitly that the computation is straightforward, and this is all that is required to compute these BPS quantities.

\section{Discussion}

In this paper we have shown that the large $N$ field theory and gravity computations of the BPS Wilson loop agree in a large class of three-dimensional $\mathcal{N}=2$ superconformal field theories with $\mathrm{AdS}_{4} \times Y_{7}$ gravity duals. In fact really this matching is a corollary of the fact that the image of the M-theory Hamiltonian $h_{M}\left(Y_{7}\right)=\left[c_{\min }, c_{\max }\right]$ is equal to the support $\left[x_{\min }, x_{\max }\right]$ of the real part of the saddle point eigenvalue distribution in the large $N$ matrix model, with the proportionality factor between the variables $x$ and $c$ given by (1.6). Moreover, the critical points of $h_{M}$, which give the loci of supersymmetric M2-branes wrapping the M-theory circle, always map under $h_{M}$ to the points at which $\rho^{\prime}(x)$ is discontinuous in the matrix model. The fact that the eigenvalue density changes behaviour every time a critical point $x_{i}$ is crossed is explained by (3.35) which relates $\rho(x)$ to the volume of a subspace of $h_{M}^{-1}(c)$ whose topology changes at the critical points $c_{i}$.

\section{Acknowledgments}

D. F. is supported by the Berrow Foundation and J. F. S. by the Royal Society. We would like to thank Chris Herzog for pointing out the results of reference [61, 62].

Open Access. This article is distributed under the terms of the Creative Commons Attribution License (CC-BY 4.0), which permits any use, distribution and reproduction in any medium, provided the original author(s) and source are credited. 


\section{References}

[1] O. Aharony, O. Bergman, D.L. Jafferis and J. Maldacena, $N=6$ superconformal Chern-Simons-matter theories, M2-branes and their gravity duals, JHEP 10 (2008) 091 [arXiv:0806.1218] [INSPIRE].

[2] M. Benna, I. Klebanov, T. Klose and M. Smedback, Superconformal Chern-Simons theories and $A d S_{4} / C F T_{3}$ correspondence, JHEP 09 (2008) 072 [arXiv:0806.1519] [INSPIRE].

[3] D. Martelli and J. Sparks, Moduli spaces of Chern-Simons quiver gauge theories and $A d S_{4} / C F T_{3}$, Phys. Rev. D 78 (2008) 126005 [arXiv:0808.0912] [InSPIRE].

[4] D.L. Jafferis and A. Tomasiello, A simple class of $N=3$ gauge/gravity duals, JHEP 10 (2008) 101 [arXiv:0808.0864] [INSPIRE].

[5] A. Hanany and A. Zaffaroni, Tilings, Chern-Simons theories and M2 branes, JHEP 10 (2008) 111 [arXiv:0808.1244] [INSPIRE].

[6] K. Ueda and M. Yamazaki, Toric Calabi-Yau four-folds dual to Chern-Simons-matter theories, JHEP 12 (2008) 045 [arXiv:0808.3768] [INSPIRE].

[7] A. Hanany, D. Vegh and A. Zaffaroni, Brane tilings and M2 branes, JHEP 03 (2009) 012 [arXiv: 0809.1440] [INSPIRE].

[8] S. Franco, A. Hanany, J. Park and D. Rodriguez-Gomez, Towards M2-brane theories for generic toric singularities, JHEP 12 (2008) 110 [arXiv:0809.3237] [INSPIRE].

[9] A. Hanany and Y.-H. He, M2-branes and quiver Chern-Simons: a taxonomic study, arXiv:0811.4044 [INSPIRE].

[10] S. Franco, I.R. Klebanov and D. Rodriguez-Gomez, M2-branes on orbifolds of the cone over $Q^{1,1,1}$, JHEP 08 (2009) 033 [arXiv:0903.3231] [INSPIRE].

[11] D.L. Jafferis, Quantum corrections to $\mathcal{N}=2$ Chern-Simons theories with flavor and their AdS $S_{4}$ duals, JHEP 08 (2013) 046 [arXiv:0911.4324] [INSPIRE].

[12] M. Aganagic, A stringy origin of M2 brane Chern-Simons theories, Nucl. Phys. B 835 (2010) 1 [arXiv: 0905.3415] [INSPIRE].

[13] F. Benini, C. Closset and S. Cremonesi, Chiral flavors and M2-branes at toric CY 4 singularities, JHEP 02 (2010) 036 [arXiv:0911.4127] [INSPIRE].

[14] A. Amariti, D. Forcella, L. Girardello and A. Mariotti, 3D Seiberg-like dualities and M2 branes, JHEP 05 (2010) 025 [arXiv: 0903.3222] [INSPIRE].

[15] J. Davey, A. Hanany, N. Mekareeya and G. Torri, Phases of M2-brane theories, JHEP 06 (2009) 025 [arXiv: 0903.3234] [INSPIRE].

[16] A. Hanany and Y.-H. He, Chern-Simons: Fano and Calabi-Yau, Adv. High Energy Phys. 2011 (2011) 204576 [arXiv:0904.1847] [INSPIRE].

[17] J. Davey, A. Hanany, N. Mekareeya and G. Torri, Higgsing M2-brane theories, JHEP 11 (2009) 028 [arXiv: 0908.4033] [INSPIRE].

[18] C. Closset and S. Cremonesi, Toric fano varieties and Chern-Simons quivers, JHEP 05 (2012) 060 [arXiv:1201.2431] [INSPIRE].

[19] O. Lunin and J.M. Maldacena, Deforming field theories with $\mathrm{U}(1) \times \mathrm{U}(1)$ global symmetry and their gravity duals, JHEP 05 (2005) 033 [hep-th/0502086] [INSPIRE]. 
[20] R. Corrado, K. Pilch and N.P. Warner, An $N=2$ supersymmetric membrane flow, Nucl. Phys. B 629 (2002) 74 [hep-th/0107220] [INSPIRE].

[21] I. Klebanov, T. Klose and A. Murugan, $A d S_{4} / C F T_{3}$ squashed, stretched and warped, JHEP 03 (2009) 140 [arXiv:0809.3773] [inSPIRE].

[22] C. Ahn and K. Woo, The gauge dual of a warped product of $A d S_{4}$ and a squashed and stretched seven-manifold, Class. Quant. Grav. 27 (2010) 035009 [arXiv:0908.2546] [INSPIRE].

[23] C. Ahn, The eleven-dimensional uplift of four-dimensional supersymmetric RG flow, J. Geom. Phys. 62 (2012) 1480 [arXiv:0910.3533] [InSPIRE].

[24] M. Gabella, D. Martelli, A. Passias and J. Sparks, The free energy of $\mathcal{N}=2$ supersymmetric $A d S_{4}$ solutions of M-theory, JHEP 10 (2011) 039 [arXiv:1107.5035] [INSPIRE].

[25] M. Gabella, D. Martelli, A. Passias and J. Sparks, $N=2$ supersymmetric AdS $S_{4}$ solutions of M-theory, arXiv:1207.3082 [INSPIRE].

[26] A. Kapustin, B. Willett and I. Yaakov, Exact results for Wilson loops in superconformal Chern-Simons theories with matter, JHEP 03 (2010) 089 [arXiv: 0909.4559] [INSPIRE].

[27] D.L. Jafferis, The exact superconformal R-symmetry extremizes Z, JHEP 05 (2012) 159 [arXiv: 1012.3210] [INSPIRE].

[28] N. Hama, K. Hosomichi and S. Lee, Notes on SUSY gauge theories on three-sphere, JHEP 03 (2011) 127 [arXiv: 1012.3512] [inSPIRE].

[29] V. Pestun, Localization of gauge theory on a four-sphere and supersymmetric Wilson loops, Commun. Math. Phys. 313 (2012) 71 [arXiv:0712.2824] [InSPIRE].

[30] C.P. Herzog, I.R. Klebanov, S.S. Pufu and T. Tesileanu, Multi-matrix models and tri-Sasaki Einstein spaces, Phys. Rev. D 83 (2011) 046001 [arXiv:1011.5487] [InSPIRE].

[31] D. Martelli and J. Sparks, The large-N limit of quiver matrix models and Sasaki-Einstein manifolds, Phys. Rev. D 84 (2011) 046008 [arXiv: 1102.5289] [INSPIRE].

[32] S. Cheon, H. Kim and N. Kim, Calculating the partition function of $N=2$ gauge theories on $S^{3}$ and AdS/CFT correspondence, JHEP 05 (2011) 134 [arXiv:1102.5565] [INSPIRE].

[33] D.L. Jafferis, I.R. Klebanov, S.S. Pufu and B.R. Safdi, Towards the F-theorem: $N=2$ field theories on the three-sphere, JHEP 06 (2011) 102 [arXiv:1103.1181] [INSPIRE].

[34] D.R. Gulotta, J. Ang and C.P. Herzog, Matrix models for supersymmetric Chern-Simons theories with an ADE classification, JHEP 01 (2012) 132 [arXiv:1111.1744] [INSPIRE].

[35] A. Amariti and M. Siani, Z extremization in chiral-like Chern Simons theories, JHEP 06 (2012) 171 [arXiv:1109.4152] [INSPIRE].

[36] A. Amariti, C. Klare and M. Siani, The large- $N$ limit of toric Chern-Simons matter theories and their duals, JHEP 10 (2012) 019 [arXiv:1111.1723] [INSPIRE].

[37] A. Amariti and S. Franco, Free energy vs. Sasaki-Einstein volume for infinite families of M2-brane theories, JHEP 09 (2012) 034 [arXiv: 1204.6040] [INSPIRE].

[38] N. Drukker and D. Trancanelli, A supermatrix model for $N=6$ super Chern-Simons-matter theory, JHEP 02 (2010) 058 [arXiv:0912.3006] [INSPIRE].

[39] M. Mariño and P. Putrov, Exact results in ABJM theory from topological strings, JHEP 06 (2010) 011 [arXiv:0912.3074] [INSPIRE]. 
[40] N. Drukker, M. Mariño and P. Putrov, From weak to strong coupling in ABJM theory, Commun. Math. Phys. 306 (2011) 511 [arXiv:1007.3837] [InSPIRE].

[41] T. Suyama, On large-N solution of ABJM theory, Nucl. Phys. B 834 (2010) 50 [arXiv: 0912.1084] [INSPIRE].

[42] J.M. Maldacena, Wilson loops in large-N field theories, Phys. Rev. Lett. 80 (1998) 4859 [hep-th/9803002] [INSPIRE].

[43] B. Assel, J. Estes and M. Yamazaki, Wilson loops in $5 D N=1$ SCFTs and AdS/CFT, arXiv:1212.1202 [INSPIRE].

[44] A. Passias, A note on supersymmetric Ad $S_{6}$ solutions of massive type IIA supergravity, JHEP 01 (2013) 113 [arXiv: 1209.3267] [INSPIRE].

[45] M. Gabella and J. Sparks, Generalized geometry in AdS/CFT and volume minimization, Nucl. Phys. B 861 (2012) 53 [arXiv:1011.4296] [InSPIRE].

[46] M. Gabella, J.P. Gauntlett, E. Palti, J. Sparks and D. Waldram, The central charge of supersymmetric AdS $S_{5}$ solutions of type IIB supergravity, Phys. Rev. Lett. 103 (2009) 051601 [arXiv: 0906.3686] [INSPIRE].

[47] M. Gabella, J.P. Gauntlett, E. Palti, J. Sparks and D. Waldram, AdS $S_{5}$ solutions of type IIB supergravity and generalized complex geometry, Commun. Math. Phys. 299 (2010) 365 [arXiv: 0906.4109] [INSPIRE].

[48] D. Martelli, J. Sparks and S.-T. Yau, The geometric dual of a-maximisation for toric Sasaki-Einstein manifolds, Commun. Math. Phys. 268 (2006) 39 [hep-th/0503183] [INSPIRE].

[49] D. Martelli, J. Sparks and S.-T. Yau, Sasaki-Einstein manifolds and volume minimisation, Commun. Math. Phys. 280 (2008) 611 [hep-th/0603021] [INSPIRE].

[50] E. Witten, Mirror manifolds and topological field theory, hep-th/9112056 [INSPIRE].

[51] A. Klemm, M. Mariño, M. Schiereck and M. Soroush, ABJM Wilson loops in the Fermi gas approach, arXiv:1207.0611 [INSPIRE].

[52] C. Closset, T.T. Dumitrescu, G. Festuccia, Z. Komargodski and N. Seiberg, Contact terms, unitarity and F-maximization in three-dimensional superconformal theories, JHEP 10 (2012) 053 [arXiv: 1205.4142] [INSPIRE].

[53] K. Becker, M. Becker and A. Strominger, Five-branes, membranes and nonperturbative string theory, Nucl. Phys. B 456 (1995) 130 [hep-th/9507158] [INSPIRE].

[54] E. Lerman, Contact toric manifolds, J. Symplectic Geom. 1 (2003) 785 [math.SG/0107201].

[55] A. Futaki, H. Ono and G. Wang, Transverse Kähler geometry of Sasaki manifolds and toric Sasaki-Einstein manifolds, J. Diff. Geom. 83 (2009) 585 [math/0607586] [INSPIRE].

[56] N. Benishti, Y.-H. He and J. Sparks, (Un)higgsing the M2-brane, JHEP 01 (2010) 067 [arXiv: 0909.4557] [INSPIRE].

[57] S. Franco et al., Gauge theories from toric geometry and brane tilings, JHEP 01 (2006) 128 [hep-th/0505211] [INSPIRE].

[58] S. Benvenuti and M. Kruczenski, From Sasaki-Einstein spaces to quivers via BPS geodesics: $L^{p, q \mid r}, J H E P 04$ (2006) 033 [hep-th/0505206] [INSPIRE]. 
[59] A. Butti, D. Forcella and A. Zaffaroni, The dual superconformal theory for $L^{p, q \mid r}$ manifolds, JHEP 09 (2005) 018 [hep-th/0505220] [INSPIRE].

[60] N. Halmagyi, K. Pilch and N.P. Warner, On supersymmetric flux solutions of M-theory, arXiv: 1207.4325 [INSPIRE].

[61] D.R. Gulotta, C.P. Herzog and S.S. Pufu, From necklace quivers to the F-theorem, operator counting and $T(\mathrm{U}(N))$, JHEP 12 (2011) 077 [arXiv: 1105.2817] [INSPIRE].

[62] D.R. Gulotta, C.P. Herzog and S.S. Pufu, Operator counting and eigenvalue distributions for $3 D$ supersymmetric gauge theories, JHEP 11 (2011) 149 [arXiv:1106.5484] [INSPIRE]. 